\title{
Aerodynamic Performance of a Transonic Turbine Blade Passage in Presence of Upstream Slot and Mateface Gap with Endwall Contouring
}

\author{
Sakshi Jain
}

Thesis submitted to the faculty of the Virginia Polytechnic Institute and State University in partial fulfillment of the requirements for the degree of

\author{
Master of Science
}

In

Mechanical Engineering

\author{
Wing F. Ng \\ Srinath V. Ekkad \\ Walter F. O'Brien Jr.
}

November 18, 2013

Blacksburg, VA

Keywords: Gas Turbines, Transonic Cascade, Secondary Flow, Upstream Purge Slot, Mateface gap, Endwall Contouring 


\title{
Aerodynamic Performance of a Transonic Turbine Blade Passage in Presence of Upstream Slot and Mateface Gap with Endwall Contouring
}

\begin{abstract}
Sakshi Jain
ABSTRACT

The present study investigates mixed out aerodynamic loss coefficient measurements for a high turning, contoured endwall passage under transonic operating conditions in presence of upstream purge slot and mateface gap. The upstream purge slot represents the gap between stator-rotor interface and the mateface gap simulates the assembly feature between adjacent airfoils in an actual high pressure turbine stage. While the performance of the mateface and upstream slot has been studied for lower Mach number, no studies exist in literature for transonic flow conditions. Experiments were performed at the Virginia Tech's linear, transonic blow down cascade facility. Measurements were carried out at design conditions (isentropic exit Mach number of 0.87 , design incidence) without and with coolant blowing. Upstream leakage flow of $1.0 \%$ coolant to mainstream mass flow ratio (MFR) was considered with the presence of mateface gap. There was no coolant blowing through the mateface gap itself. Cascade exit pressure measurements were carried out using a 5-hole probe traverse at a plane 1.0Cax downstream of the trailing edge for a planar geometry and two contoured endwalls. Spanwise measurements were performed to complete the entire 2D loss plane from endwall to midspan, which were used to plot pitchwise averaged losses for different span locations and loss contours for the passage. Results reveal significant reduction in aerodynamic losses using the contoured endwalls due to the modification of flow physics compared to a non contoured planar endwall.
\end{abstract}




\section{Acknowledgements}

This endeavor would not have been possible without the support and guidance of many individuals, whom I would like to acknowledge here. I wish to thank both Dr. Srinath Ekkad and $\mathrm{Dr}$. Wing $\mathrm{Ng}$ for their guidance throughout the project, and for the duration of my Masters here at Virginia Tech. Their critiques and feedback have driven the improvement in my work, and enhancement in my technical understanding. In the same vein, I wish to acknowledge the inputs from Andrew Lohaus and Frazad Taremi from Siemens. Their able direction has helped me take decisions to make the project more meaningful. The work has been funded by Siemens Inc and I wish to thank them for making this possible. A special thanks to Dr. O'Brien to whom I owe much of my knowledge in the subject matter of turbomachinery, and l've learnt immensely from my discussions with him in and beyond the classroom.

I would like to thank my lab mates, particularly Hunter Guilliams, Bonaventure Nunes, Jaideep Pandit, David Gomez Ramirez, Shreyas Srinivasan and friends Deepu Dilip and Vivek Kumar. The challenging project can take its toll, and it is the presence of great friends like these whose support and friendship makes the experience worthwhile.

I'm grateful to Dorian Blot for his immense patience as I learnt the necessary skills, which enhanced my efficacy as an investigator on the project. I am deeply indebted to Arnab Roy, for it is his constant leadership, guidance and friendship which has made this project possible.

Lastly, I wish to acknowledge the support of my family, particularly my mother. Despite the geographical distance, it is their faith which motivates me each day to deliver my best. 


\section{CONTENTS}

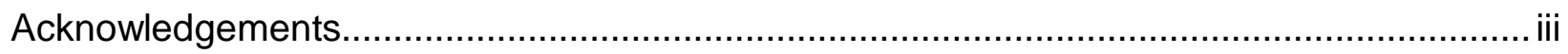

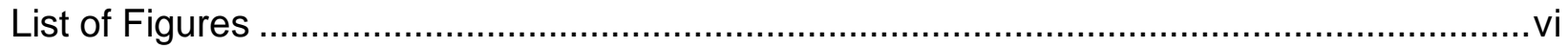

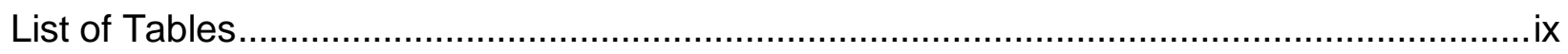

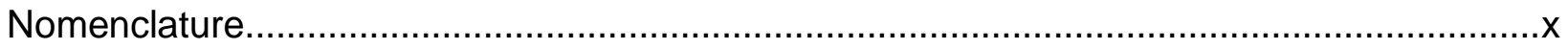

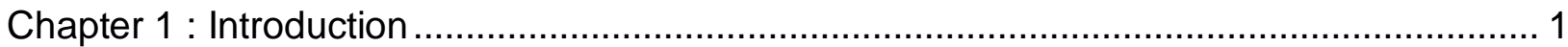

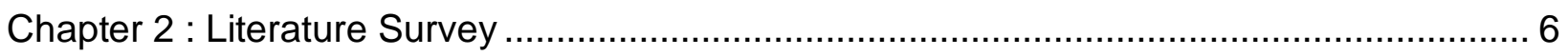

Chapter 3 : Test Facility, Experimental Set Up, Data Processing ..................................... 10

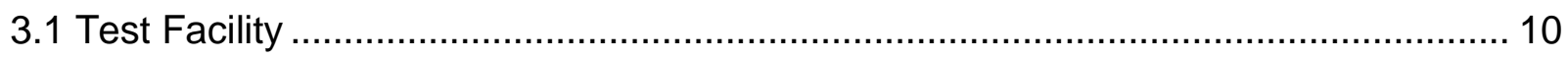

3.1.1 Transonic Wind Tunnel.............................................................................. 10

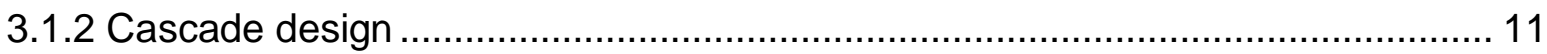

Upstream leakage slot \& Mateface Gap design.......................................................... 12

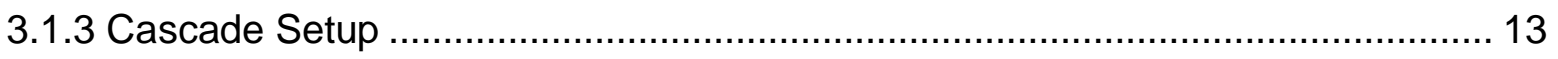

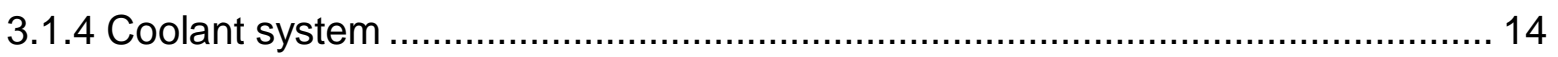

3.2 Aerodynamic Measurement Technique ............................................................. 16

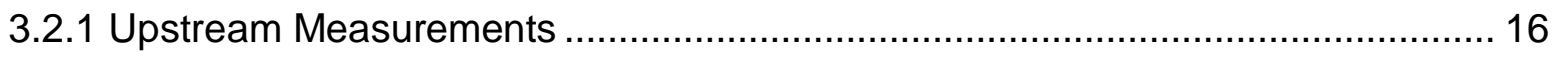

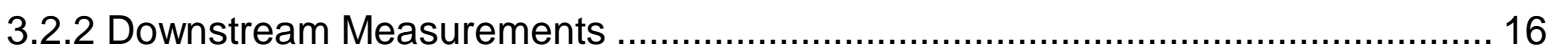

3.3 Data Reduction \& Loss Coefficient Calculation ........................................................ 20

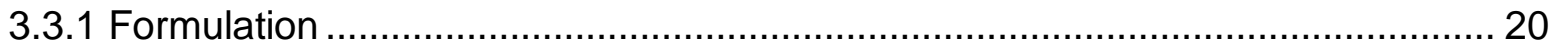

3.4 Uncertainty in Loss Coefficient Measurements..................................................... 22

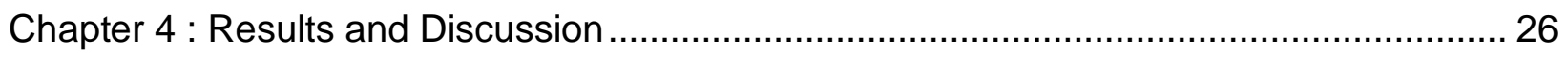

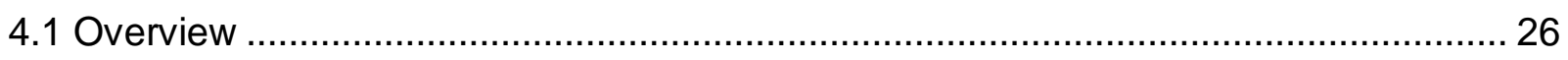

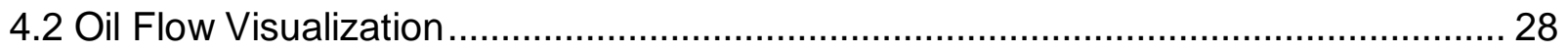

4.3 Effect of Mateface Gap .................................................................................... 34

4.4 Effect of coolant injection ............................................................................... 36 


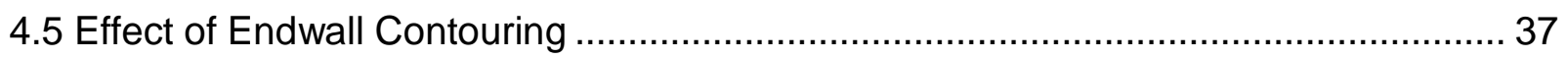

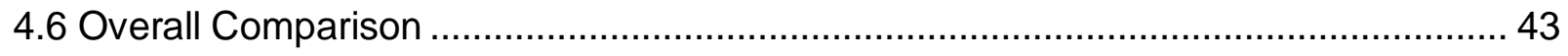

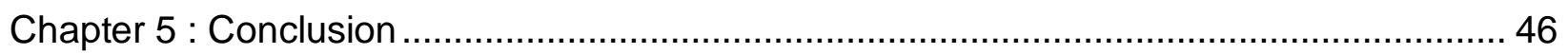

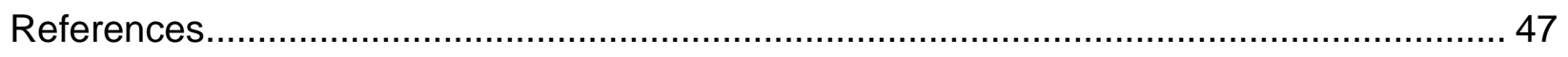




\section{List of Figures}

Figure 1: Electricity Generation Projection[1] (Figure 6 International Energy Outlook 2011

Report Number: DOE/EIA-0484.Used under fair use, 2013) .......................................... 1

Figure 2 : Secondary Flow Models a) Langston [7] b) Sharma \& Butler [9] c) Goldstein \&

Spores [10] d) Takeishi et al [11] ........................................................................... 3

Figure 3 : Saddle point and Separation Lines in Secondary Flow (Figure 4 S. Acharya,2000,

"Endwall Cooling With Endwall Contouring and Leading Edge Fillet," Semi-annual Report

Submited to UTSR, South Carolina, Project No. 02-01-SR098, June2003-December

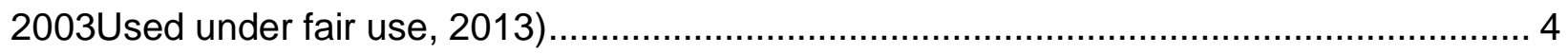

Figure 4: Virginia Tech Transonic Wind Tunnel Facility ................................................ 11

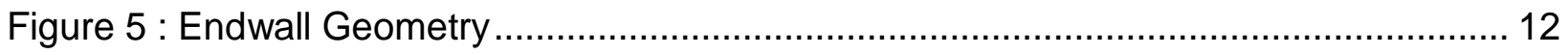

Figure 6 : Upstream Slot \& Mateface Gap Design ............................................................ 13

Figure 7 : Cascade Setup (a) CAD Model depicting location of turbulence grids, (b) Actual picture of the cascade with baseline endwall, indicating location of headboard and tailboard

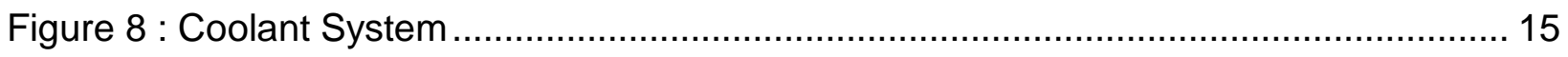

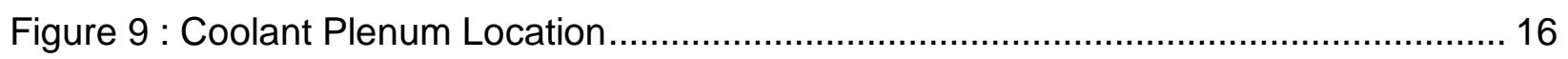

Figure 10 : Pressure Probe locations ........................................................................... 17

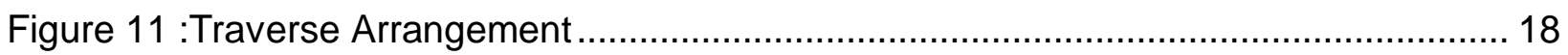

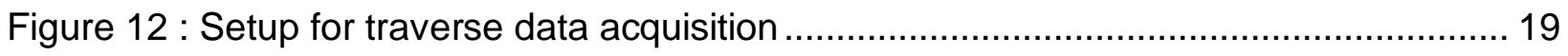

Figure 13 :Midspan airfoil loading from CFD and experiments [21] (Figure 5 Effect of Endwall Contouring on a Transonic Turbine Blade Passage:Part 1 - Aerodynamic Performance", ASME Paper No. GT2012-68425. Used under fair use, 2013) ................... 19

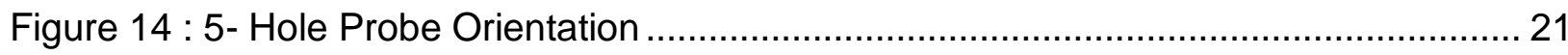


Figure 15 : Uncertainty Correlation with loss Coefficient for $\mathrm{Ma}=0.87 \ldots \ldots \ldots \ldots \ldots \ldots \ldots \ldots \ldots \ldots . . .25$

Figure 16 : Parameters for Aerodynamic Performance Study ........................................... 26

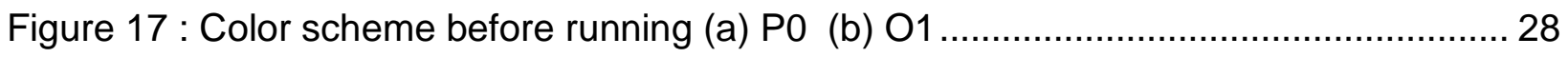

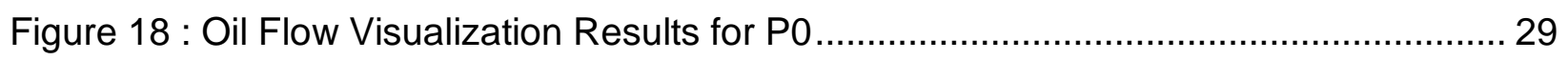

Figure 19 : Oil Flow Visualization for OPT Contour.......................................................... 31

Figure 20 : Comparison between oil flow passage view (a) Case P0 (b) Case O1.............. 33

Figure 21 : Comparison between oil flow TE view (a) Case P0 (b) Case O1 ....................... 33

Figure 22 : Effect of Addition of Mateface with No Coolant Injection- Pitchwise Averaged

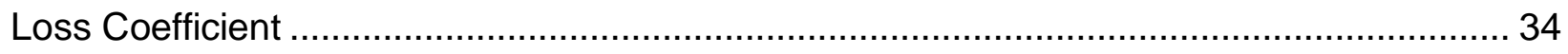

Figure 23 : Effect of Mateface Gap Addition - Loss Contours.............................................. 35

Figure 24 : Effect of Coolant addition (a) Planar (b) Aero Contour (c) OPT contour -

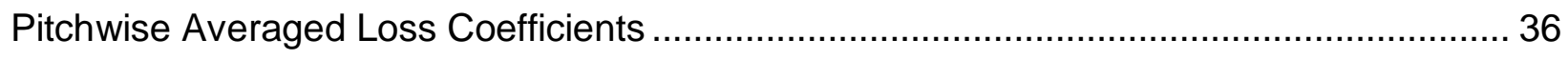

Figure 25 : Effect of Coolant Injection through Upstream Purge Slot in Planar Endwall Loss

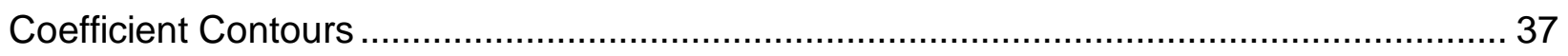

Figure 26 : Effect of Endwall Contouring - Pitchwise Averaged Loss Coefficients (a) Without

Coolant (b) With 1\% MFR through upstream slot.............................................................. 38

Figure 27 : Effect of Endwall Contouring with and without Coolant Injection Loss Coefficient

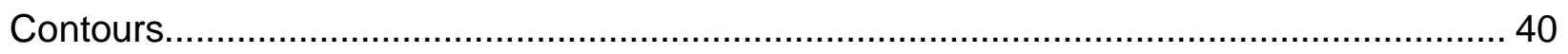

Figure 28 : Comparison of Secondary Flow between Planar endwall and Aero contour [25]

(Figure 23 Panchal, K., 2011, "Development of a Robust Numerical Optimization Methodology for Turbine Endwalls and Effect of Endwall Contouring on Turbine Passage Performance,"Doctor of Philosophy, Virginia Polytechnic Institute and State University, Blacksburg, Virginia. Used under fair use, 2013) ……............................................... 41

Figure 29 : Near Endwall Exit Mach\# pitchwise distribution for P1,A1 \& O1 ....................... 42 


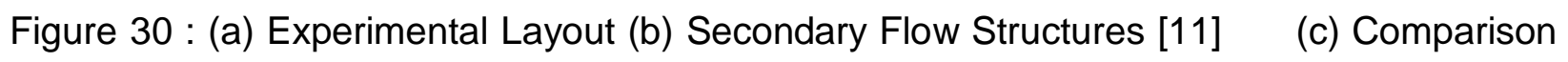
between Loss contours and Passage vortex growth for Planar No Mateface (P0), Planar with Mateface (P1), Contoured with Mateface (C1) in absence of purge cooling Loss

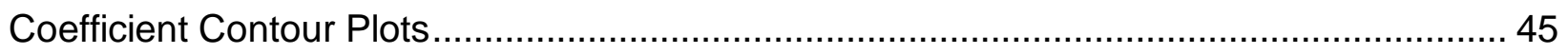




\section{List of Tables}

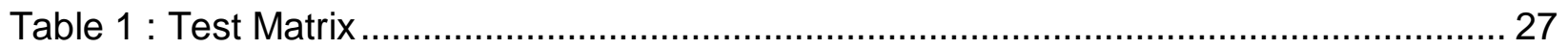

Table 2 : Loss Coefficient Comparison ......................................................................... 44 


\section{Nomenclature}

\begin{tabular}{|c|c|}
\hline$C_{a x}$ & Axial chord length \\
\hline$C_{p}$ & The specific heat capacity at a constant pressure \\
\hline$C_{v}$ & The specific heat capacity at a constant volume \\
\hline$H T C, h$ & Heat transfer coefficient \\
\hline$M_{\text {iso }}$ & Isentropic Mach number $\boldsymbol{M}_{\text {iso }}=\sqrt{\left[\left(\frac{p_{01}}{p_{s 2}}\right)^{\frac{\gamma-1}{\gamma}}-1\right] \frac{2}{\gamma-1}}$ \\
\hline$M F R$ & $\begin{array}{l}\text { Mass flow ratio = coolant mass flow rate per passage/ } \\
\text { mainstream mass flow rate per passage }\end{array}$ \\
\hline$p_{0}$ & Local stagnation pressure \\
\hline $\boldsymbol{p}_{s}$ & Local static pressure \\
\hline$p_{\text {s ex }}$ & Exit Static Pressure \\
\hline$p_{s} \operatorname{ex}(a)$ & Area averaged Exit Static Pressure \\
\hline$P t_{e x}$ & Exit total pressure \\
\hline$P t_{e x(m)}$ & Mass Averaged Exit total pressure \\
\hline$P t_{i n}$ & $\begin{array}{l}\text { Inlet Total Pressure measured } 0.45 \mathrm{C}_{\mathrm{ax}} \text { upstream of the } \\
\text { cascade }\end{array}$ \\
\hline PS & Pressure Surface \\
\hline $\mathbf{p}$ & Pitch \\
\hline$s$ & Span \\
\hline SS & Suction Surface \\
\hline$T_{0}$ & Stagnation temperature \\
\hline$T, T_{s}$ & Static temperature \\
\hline$T_{t}$ & Total temperature \\
\hline
\end{tabular}




$\begin{array}{ll}\boldsymbol{T I T} & \text { Turbine Inlet Temperature } \\ \boldsymbol{x} & \text { Pitchwise Coordinate } \\ \mathbf{y} & \text { Spanwise Coordinate } \\ \boldsymbol{\gamma} & \text { Heat Capacity Ratio } \frac{c_{p}}{c_{v}} \\ \boldsymbol{\rho} & \text { Density } \\ \boldsymbol{\omega} & \text { Local Loss coefficient }=\frac{\boldsymbol{p}_{t, \boldsymbol{i n}}-\boldsymbol{p}_{t, \boldsymbol{e x i t}}}{\boldsymbol{p}_{t, \boldsymbol{e x i t}}-\boldsymbol{p}_{\boldsymbol{s}, \boldsymbol{e x i t}}} \\ & \\ \boldsymbol{\omega}_{\text {overall }} & \text { Overall Loss coefficient }=\frac{\boldsymbol{p}_{t, \boldsymbol{i n}}-\boldsymbol{p}_{t, \boldsymbol{e x}}(m)}{\boldsymbol{p}_{t, \boldsymbol{e x}(\boldsymbol{m})}-\boldsymbol{p}_{s, \boldsymbol{e x}(\boldsymbol{a})}}\end{array}$




\section{Chapter 1 : Introduction}

Electricity is the world's fastest-growing form of end-use energy consumption As per the projection by US Energy Information Administration[1], the net electricity generation worldwide rises by 2.3 percent per year on average from 2008 to 2035, while total world energy demand grows by 1.6 percent per year. Coal provides the largest share of world electricity generation, although its share is expected to fall. Renewables are projected to grow from 19 percent to 23 percent but they tend to be less robust than conventional thermal sources owing to inherent unpredictability. Much of this growth is expected from industrial gas turbines They can operate at very high efficiency of about $60 \%$ in combined cycle mode in gas turbine based power generation[2], at very low emissions as compared to the coal fired plants.

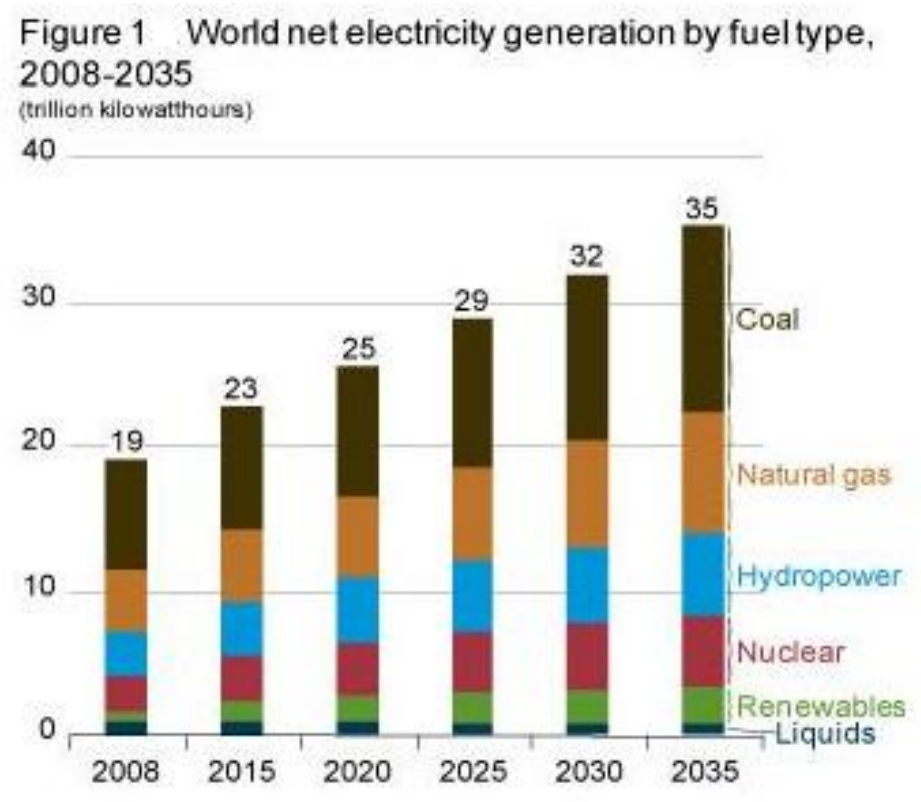

Figure 1 : Electricity Generation Projection[1] (Figure 6 International Energy

Outlook 2011 Report Number: DOE/EIA-0484.Used under fair use, 2013) 
Further development continues in gas turbine, with emphasis not only on natural gas fired plants but also with the potential of utilization in Integrated Gasification Combined Cycle units. The DOE-H2 Program which the Department of Energy has cosponsored with Siemens is an initiative in that direction and one of the thrust areas was to aim for improved thermal and aerodynamic performance in gas turbines.

A turbine flow passage is bound by suction side and pressure side of adjoining blades, and hence an inherent pressure gradient exists in the passage. This pressure gradient has the maximum impact on the low momentum near the endwall. The upstream boundary layer ingested in a turbine blade row mixes with this low momentum flow near the endwall. The subsequent flow interaction gives rise to secondary flow vortices, which typically contributes to about a third of the total losses in turbines[4]. Turbine airfoils are typically made and assembled in segments with provision for thermal expansion which results in mateface gaps. Additionally the interface between a stator and rotor creates an upstream slot which presents a risk of ingress of hot gases which may result in thermal stresses. An optimum amount of cooling air is bled off from intermediate stages of the compressor and injected through the upstream slot thus preventing hot gas ingress. This slot flow also affects the endwall flow characteristics. Moreover, turbines are being designed with fewer blades, and the resulting increased blade loading leads to greater secondary losses. All these features contribute to the complexity in secondary flows. These secondary flows affect both aerodynamic performance and heat transfer mitigation. Thus, secondary flows have gained prominence, with active research dedicated to understanding and reducing these losses to enhance aerodynamic efficiency. 


\section{Secondary Flow Physics}

The first of the models for secondary flows in turbines were described by Hawthorne [4] in 1955 with regards to the nature of the components of vorticity in the direction of flow.

Klein [5]showed for the first time the model of the cascade passage vortex. In the coming years, studies were conducted by Sjolander [6], Langston [7] and many more, which were finally summarized by Sieverding [8] in his comprehensive review paper. These were further followed by Sharma and Butler [9], Goldstein and Spores [10], Takeishi et al [11].

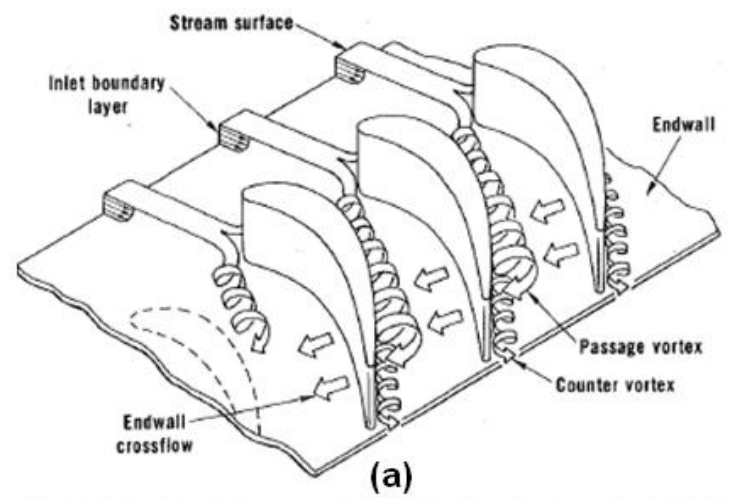

Figure 1 Langston, L. S.,1980, "Crossflows in a turbine cascade passage."American Society of Mechanical Engineers, Gas Turbine Conference and Products Show, New Orleans, La. Used under fair use, 2013.

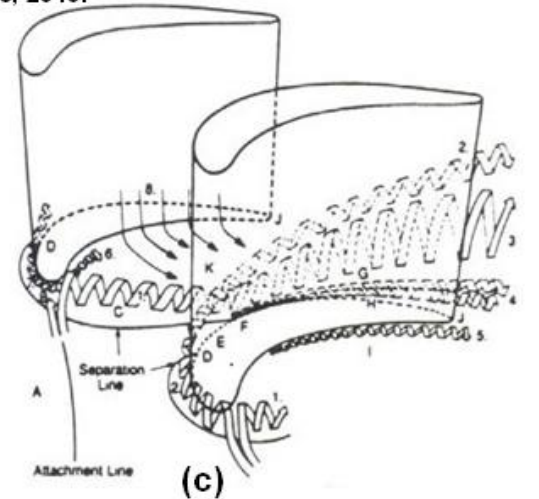

Figure 2 Goldstein, R. J., Spores, R. A., 1988, "Turbulent transport onthe endwall in the region between adjacent turbine blades." Journal of Heat Transfer (Transcations of the ASME, Series C);(United States) 110.4A. Used under fair use, 2013.

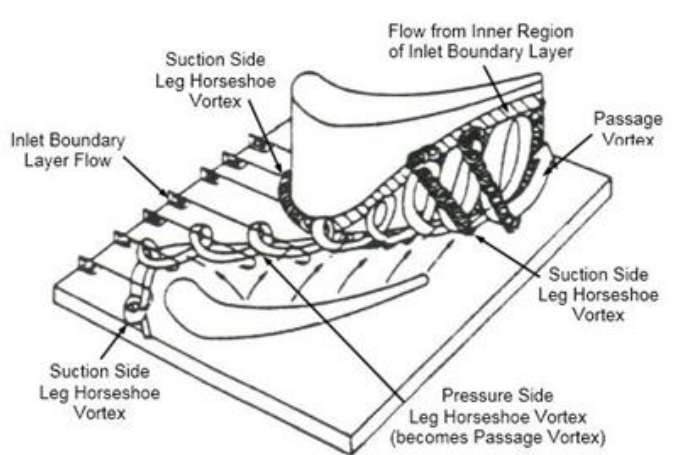

(b)

Figure 2 Sharma, O. P., Butler T. L., 1987, "Predictions of endwall losses and secondary flows in axial flow turbine cascades." Journal of turbomachinery 109.2: 229-236. Used under fair use, 2013.

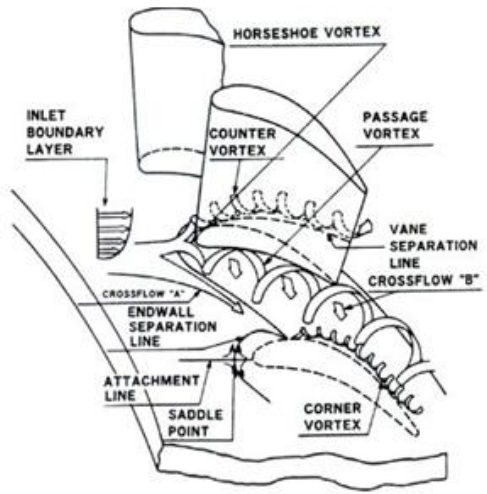

(d)

Figure 1 Takeishi, K., 1989, "An experimental study of heat transfer and film cooling on low aspect ratio turbine nozzles." American Society of Mechanical Engineers. Used under fair use, 2013.

\section{Figure 2 : Secondary Flow Models a) Langston [7] b) Sharma \& Butler [9] c) Goldstein \& Spores [10] d) Takeishi et al [11]}




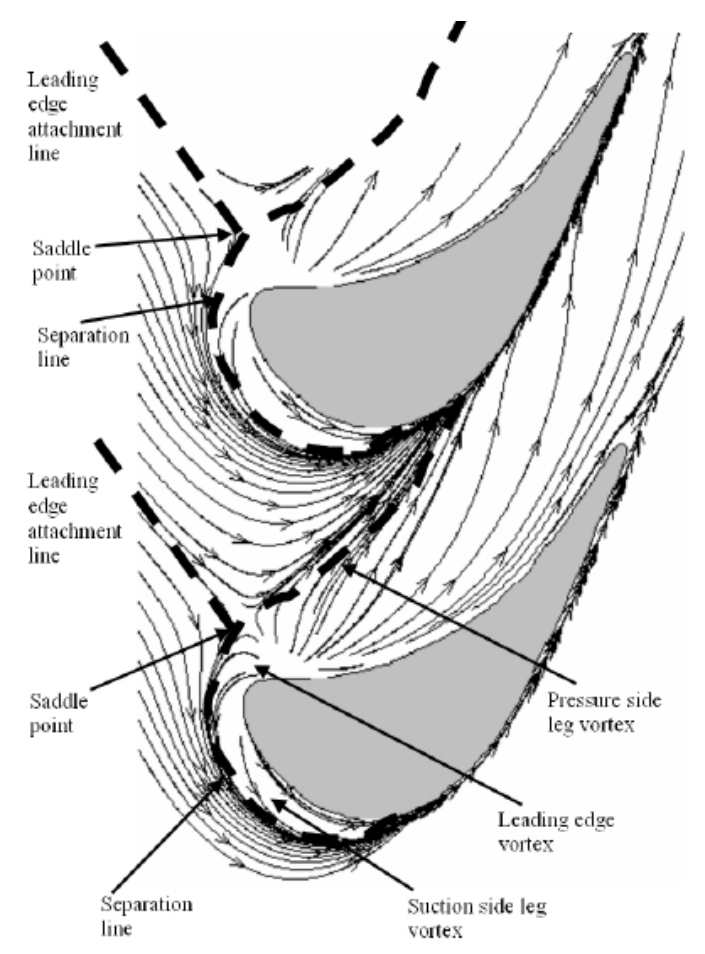

Figure 3 : Saddle point and Separation Lines in Secondary Flow (Figure 4 S. Acharya,2000, "Endwall Cooling With Endwall Contouring and Leading Edge Fillet," Semi-annual Report Submited to UTSR, South Carolina, Project No. 0201-SR098, June2003-December 2003Used under fair use, 2013)

They all came up with detailed explanation of the unique flow features near the endwall, which are caused by the ingestion of the upstream boundary layer. This layer bifurcates at the leading edge of the airfoil, and this point of separation is called the saddle point (refer Figure 3) The pressure rise in the boundary layer is less than in the freestream flow, which causes the roll up of flow and formation of a vortex called the horseshoe vortex. There maybe a small region of separation just beyond the saddle point on the pressure side, after which the flow reattaches. The horseshoe vortex divides into two distinct sections, which travel along the suction side and pressure side separately and hence are named as the suction side leg and pressure side leg respectively. Both these legs have an opposite sense of rotation to each other. They also induce leading edge vortices on both the suction and 
pressure side respectively. The pressure side leg is influenced by the pressure gradient caused by the turning of flow around the blade, \& migrates towards suction side of the adjacent airfoil. In the process it entrains the low velocity flow near the endwall to form the passage vortex, driven by the spanwise pressure gradient. This causes the passage vortex to lift off from the endwall as it meets the suction side of the adjacent airfoil. The suction leg of the horseshoe vortex, interacts with the passage vortex and wraps itself around it. This passage vortex grows in size and strength as it moves along the suction side, and is the biggest contributor to losses. Halfway down the passages, corner vortices are also induced on suction and pressure side of the airfoil due to interaction between the endwall and the boundary layer at the blade trailing edges. In case of highly loaded airfoils, the corner vortices also exist both on suction and pressure side, which tend to be difficult to visualize experimentally. 


\section{Chapter 2 : Literature Survey}

Blair [13] conducted experiments to determine the film cooling effectiveness and convective heat transfer coefficient distributions on the endwall of a turbine vane passage which simulated the passage geometry and upstream cooling slot geometry of a typical first stage turbine. He was one of the earliest to suggest the possible impact of the coolant on the flow. He concluded that the vane passage endwall heat transfer is strongly influenced by the existence of the large vortex located in the corner between the endwall and the suction surfaces but the influence of corner vortex is relatively unaffected by the location of endwall boundary layer transition or the existence of a coolant film injected upstream of the vane leading-edge line.

Blanco et al [14] also looked into the effects of a backward and forward facing step with and without leakage flow and found that the forward facing step produced stronger endwall flows compared to the backward facing step. However, the addition of leakage flow causes the loss cores for the corner and passage vortex to increase, while migrating closer towards the midspan.

Hada et al [15] conducted a computational study of a film-cooled endwall. The endwall design consisted of both an upstream slot, representing the combustor-turbine junction, and a midpassage slot, representing the mating between the adjacent vanes. While, the focus of this study was on comparing adiabatic effectiveness levels on the endwall with varying leakage flow rates and gap widths, from an aerodynamic performance point of view an important conclusion that emerged was that the secondary flow features were modified, as the passage vortex was clearly seen in the case without the mateface gap, while there was no clearly defined passage vortex where the midpassage gap was present due to the strong ingestion. 
Piggush et al [16] evaluated the use of endwall contouring in a first stage stator cascade with various steps, gaps, and leakage flows to assess their importance in this contoured passage, while also looking at features associated with the mateface (referred to as slashface in the study) on the vane platform .They documented the component misalignment and injected and leakage flow effects on the aerodynamic losses within a passage that has one contoured and one straight endwall, similar to the current study. However their study was carried out at a low speed facility with a much lower exit mach number. They concluded that higher blowing rates through the slashface gap increased the losses. However, it was also observed that the conclusion would be affected by the experimental setup since the case with no blowing had the gaps covered. Further studies[17] were conducted by them at varying mass flow rates for both the mateface gap and upstream slot, and they reported increased losses with increased mass flow rates.

Yamao et al [18] studied first stage stationary vanes for high pressure turbines for evaluating both heat transfer and aerodynamic performance for variation in sealing air flow rates. They did not find a significant impact of sealing air flow on passage loss.

Reid et al [19] investigated the effect of leakage flow through the chord-wise inter platform gaps in aeroengine nozzle guide vanes (NGV's) on turbine performance through efficiency measurements and NGV exit area traverse data from a low-speed research turbine. They also compared results with steady-state simulations with particular emphasis laid on the physics of the mainstream/leakage interaction and the loss generation. The conclusion was that this leakage flow can have a significant impact on turbine performance. However, below a threshold leakage fraction this penalty does not rise with increasing leakage flow rate. In other words, the presence of the mateface/leakage gap in itself caused increased losses, and addition of coolant through the mateface did not add significantly to the losses. 
All these studies were conducted at low Mach numbers. One somewhat similar study that was conducted at transonic conditions like the present work is by Kost et al [20] They looked at aerodynamic performance in presence of upstream slot and cooling holes. They compared losses for case with no coolant injection, with coolant injection from slot only, and the case with combination coolant blowing from upstream slot as well as cooling holes. The differences in energy loss existed mainly in the region $0-10 \%$ of the span. The overall area averaged loss value increased slightly with addition of coolant.

The aerodynamic performance at transonic speeds for a linear cascade with a diverging endwall at design and off design conditions was published by Abraham et al. [21]. In their work, they measured the loss coefficients at both 0.1-Cax and 1.0-Cax from the trailing edge of an airfoil. They concluded that the secondary loses would increase as a result of increasing the pitch by $25 \%$ using the same blade profile. Blot et al [22] utilized a similar setup to investigate aerodynamic losses for the cascade in presence of an upstream purge slot. They found that the upstream slot significantly affects the secondary flow. However, coolant blowing through the slot does not have a significant impact, indicating that the geometric disruption caused by the slot is the dominant factor.

The present study is an extension of the work presented by Abraham et al [21] and Blot et al [22] to incorporate effects of an upstream purge slot and mateface gap on the same linear cascade set-up. The measurements were carried out for a diverging planar endwall (P) as well as two contoured endwalls which shall be henceforth referred to as Aero Contour (A) and OPT1 (O) . Aero Contour has been tested previously by Abraham et al [21] and Blot et al [22]. A new contour design geometry was also tested this year, which is being referred to here as the OPT1 endwall. The effect of coolant injection was also investigated, for which $1 \%$ MFR was injected through the upstream purge slot. No coolant was injected through the mateface gap in this study. All measurements were made at design exit Mach 
number (0.87) and design incidence angle. The aerodynamic losses for the case without any mateface gap have also been presented here to highlight the influence of the slot itself and then the effects of coolant injection and endwall geometry are discussed.

For this study, the measurements were made at $1.0 \mathrm{Cax}$. The losses tend to be mixed out and the individual flow features are difficult to distinguish. The periodicity of flow deteriorates somewhat since the flow starts turning towards the exit of the cascade. However, the location was chosen since it yields a better estimate of reduction of losses due to endwall contouring, as reported by Abraham et al [21] where they saw a $3 \%$ reduction based on the measurements at $0.1 \mathrm{Cax}$ which magnified to $17 \%$ at $1.0 \mathrm{Cax}$. While the contours, may not be very reliable indicators in pinpointing specific flow features on the endwall, the results can offer insight with regards to a general trend with respect to the relative impact with geometry variation and cooling features. 


\section{Chapter 3 : Test Facility, Experimental Set Up, Data Processing}

\subsection{Test Facility}

\subsubsection{Transonic Wind Tunnel}

A schematic of the Virginia Tech transonic linear cascade wind tunnel is presented in Figure 4. The wind tunnel is a blow down facility and has provision for conducting heated runs. For aerodynamic testing, heating was not required and hence only the first and the second valve(shown in figure) were kept open, and the third was closed. The control parameter for this facility is the inlet total pressure, which is maintained for about 20 seconds using a butterfly valve, which gives a sufficient sampling window at constant Mach number. The Mach number is fixed by regulating the supply total pressure.

$$
M_{\text {isentropic }}=\sqrt{\left(\left[\frac{P_{0 \text { in }}}{P_{\text {sout }}}\right]^{\frac{\gamma-1}{\gamma}}-1\right) * \frac{2}{\gamma-1}} \quad \text { Equation [1] }
$$

The isentropic cascade exit Mach numbers in this facility can be varied from about 0.7 1.2. For this study, the exit isentropic Mach number was fixed at 0.87 for all measurements.

The air is fed from storage tanks which are charged by a dedicated compressor to maintain an uninterrupted air supply. The facility is also equipped with safety measures in the form of a shut off valve which cuts the access to the wind tunnel once the inlet total pressure to the test section exceeds $18 \mathrm{psi}$. 


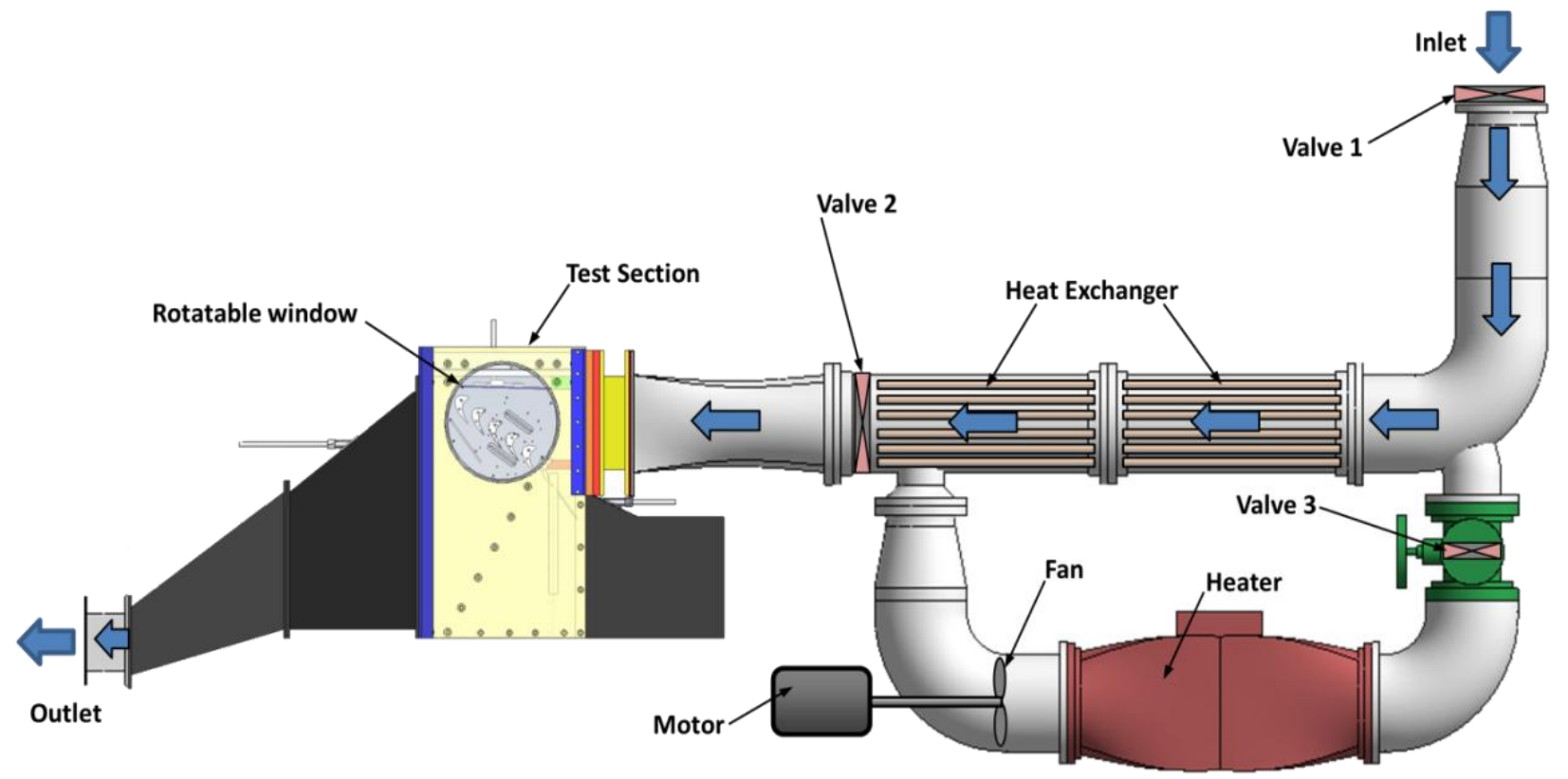

Figure 4: Virginia Tech Transonic Wind Tunnel Facility

\subsubsection{Cascade design}

\section{Endwall Design}

The airfoils are manufactured using a 3-D printing machine along with the endwall, the designs for which were provided by Siemens Energy Inc. These are then sanded for a smooth finish. To obtain the required leading edge loading in a quasi-2D cascade and to simulate a near endwall loading and required inlet-to-exit Mach number ratio that is representative to that encountered in a real engine, one endwall is diverging from inlet to exit at a $13^{\circ}$ angle. This leads to about $16 \%$ increase in airfoil span in the axial direction, from inlet to exit of the cascade. There were four endwall geometries that were tested in this study. One is a planar endwall without mateface, and the others all have both mateface and upstream slot. Two of them are contoured endwalls (Figure 5). The locations of peak and 
valley regions are highlighted for the contoured endwall. All designs for the endwalls were provided by Siemens.
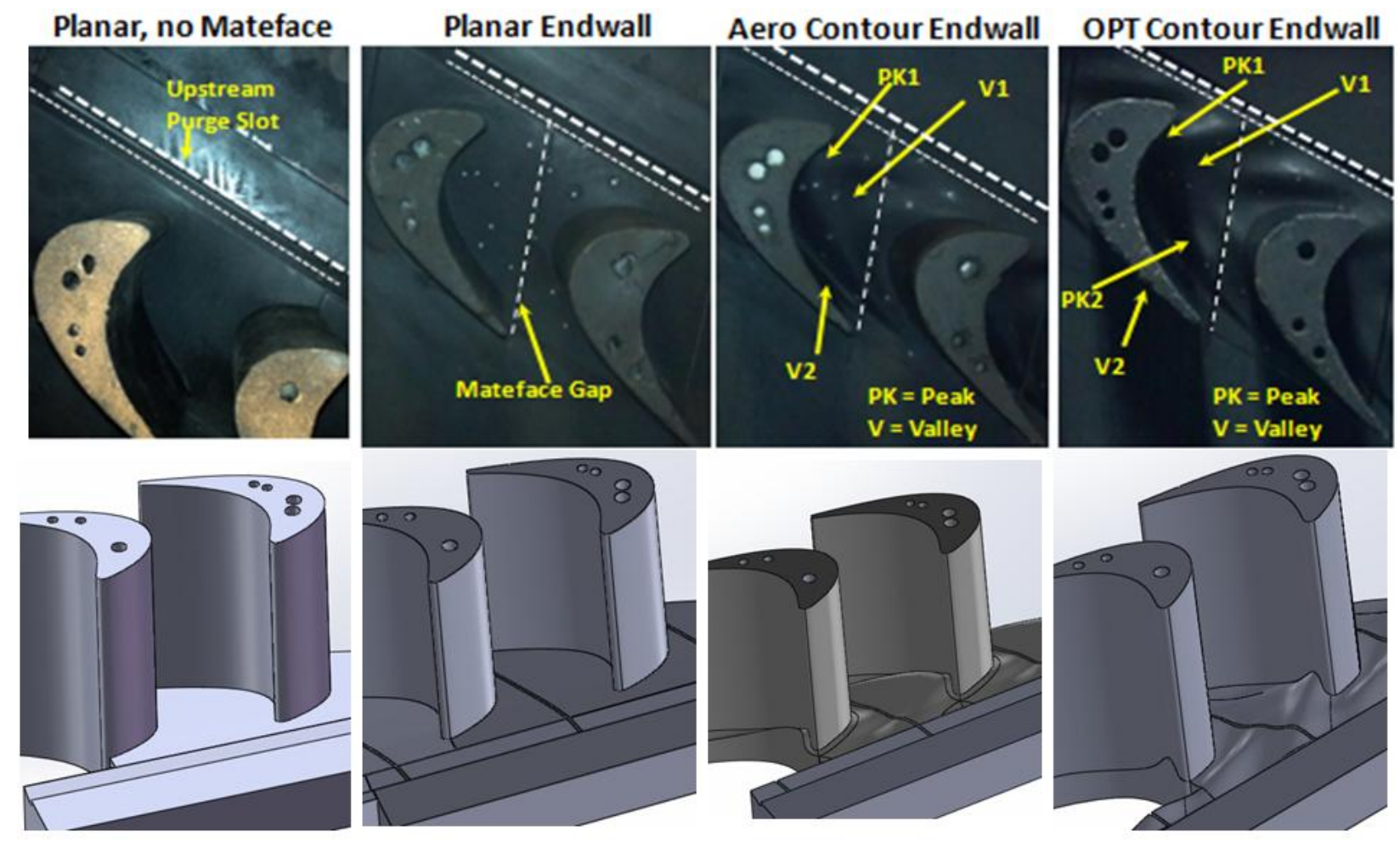

Figure 5 : Endwall Geometry

\section{Upstream leakage slot \& Mateface Gap design}

The upstream purge slot was first tested by Blot et al [22] and as reported, it is located 0.3-Cax upstream of the leading edge of airfoil. Straight parallel vanes were used in order to obtain uniform exit flow as the coolant exits the slot and interacts with the mainstream flow. To maintain uniformity of flow around the passage of interest, the slot was extended to cover $\sim 2.5$ pitch distance. The mateface slot was incorporated for this year's study and has been shown in Figure 4. It simulates the interface between two rotor blade sections. It starts from the upstream slot and extends through $0.1-\mathrm{Cax}$ after the trailing edge. The gap width is about 0.03-Cax and coolant is fed through a series of holes that 
eject coolant normally to the gap walls. However, for this study only upstream purge slot cooling was utilized.

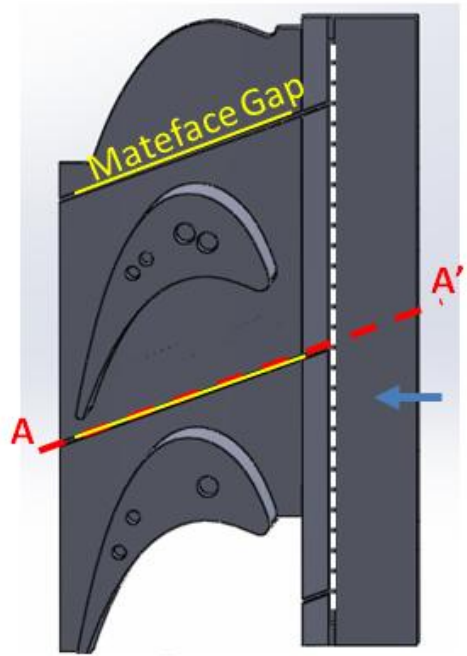

Front

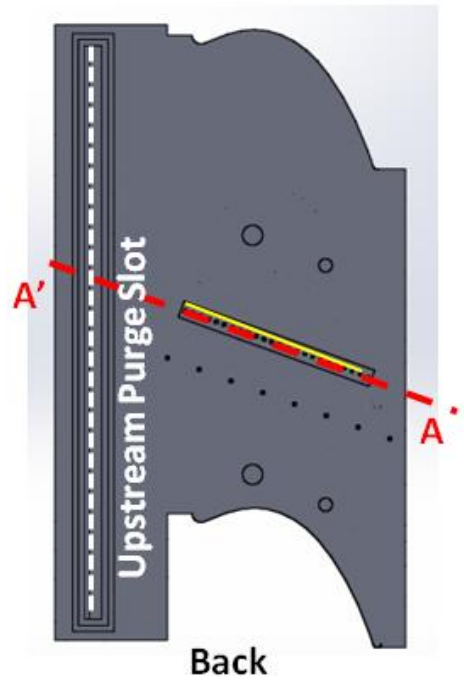

Back

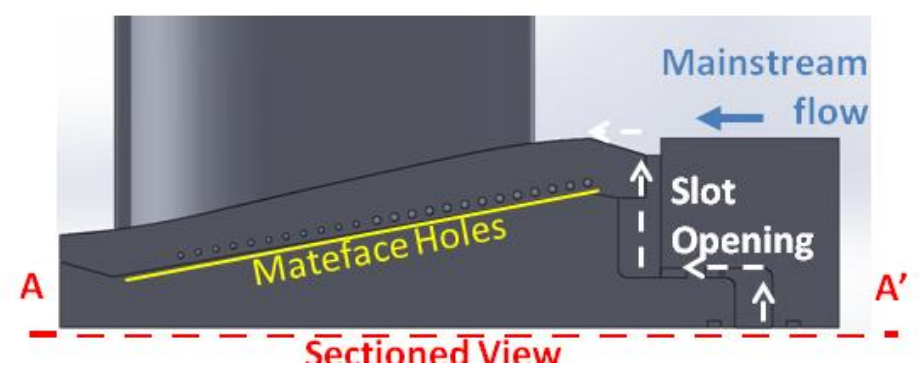

Figure 6 : Upstream Slot \& Mateface Gap Design

\subsubsection{Cascade Setup}

The test section schematic is shown in Figure 7. Since the study is a continuation from the works of Abraham et al [21]. and Blot et al. [22], an identical test section cascade geometry is used. It is a linear cascade consisting of 5 airfoils (total 4 passages), where the passage of interest is between the 3rd and 4th airfoil from the top. For this study, the cascade angle was kept constant at the design value. The flow periodicity is maintained by the use of a headboard upstream and two tailboards downstream of the cascade to guide the flow evenly(Figure 7). The turbulence grids are located at 5.5-Cax from the center 
airfoil which produces about $8 \%$ inlet free stream turbulence intensity measured at 0.5 Cax upstream of the leading edge. The experiments utilize the upstream total pressure as reference for loss measurements, which is measured at a location 0.45 Cax upstream of the cascade. This slot is also used for measuring the upstream boundary layer thickness, and the turbulence intensity of the cascade. Downstream measurements can be made at both $0.1 \mathrm{C}_{\mathrm{ax}}$ and $1.0 \mathrm{C}_{\mathrm{ax}}$, and the latter was utilized in this study.

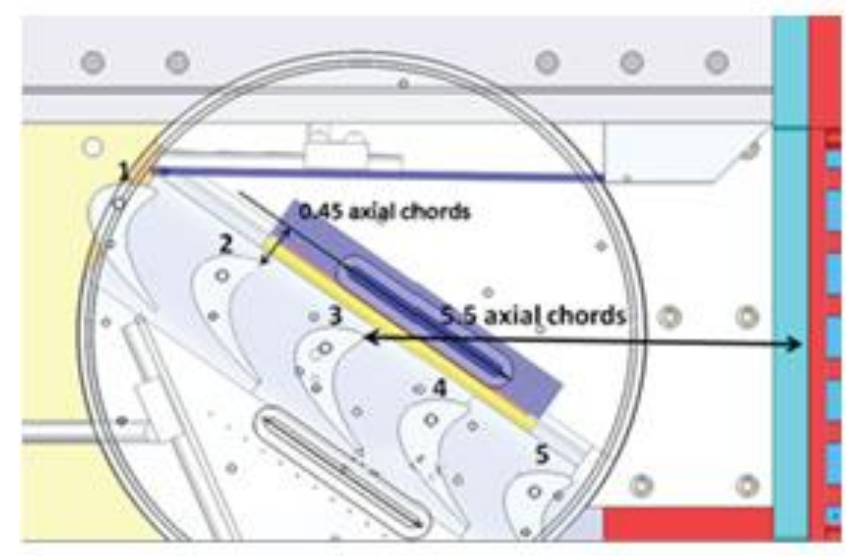

(a)

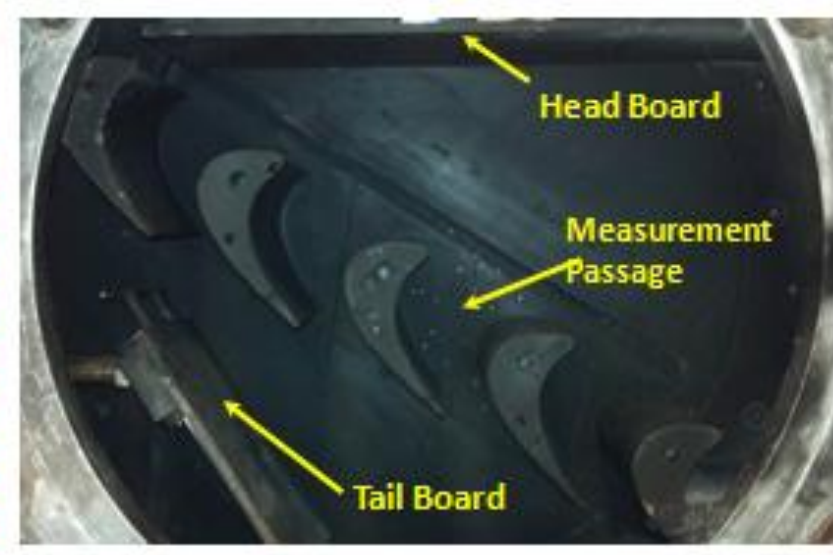

(b)

Figure 7 : Cascade Setup (a) CAD Model depicting location of turbulence grids, (b) Actual picture of the cascade with baseline endwall, indicating location of headboard and tailboard

\subsection{4 $\underline{\text { Coolant system }}$}

Figure 8 shows the schematic of the coolant supply mechanism. It consists of a high pressure compressed air storage tank which was charged using an external compressor and this supplied the required coolant. The setup utilized a pair of solenoid valve which would switch the coolant supply into the test section based on when the mainstream flow was introduced into the cascade. It was also instrumented for measuring mass flow rate and 
temperature during the run. The heater/chiller sections were not utilized for aerodynamic measurements as coolant at ambient temperature was used.
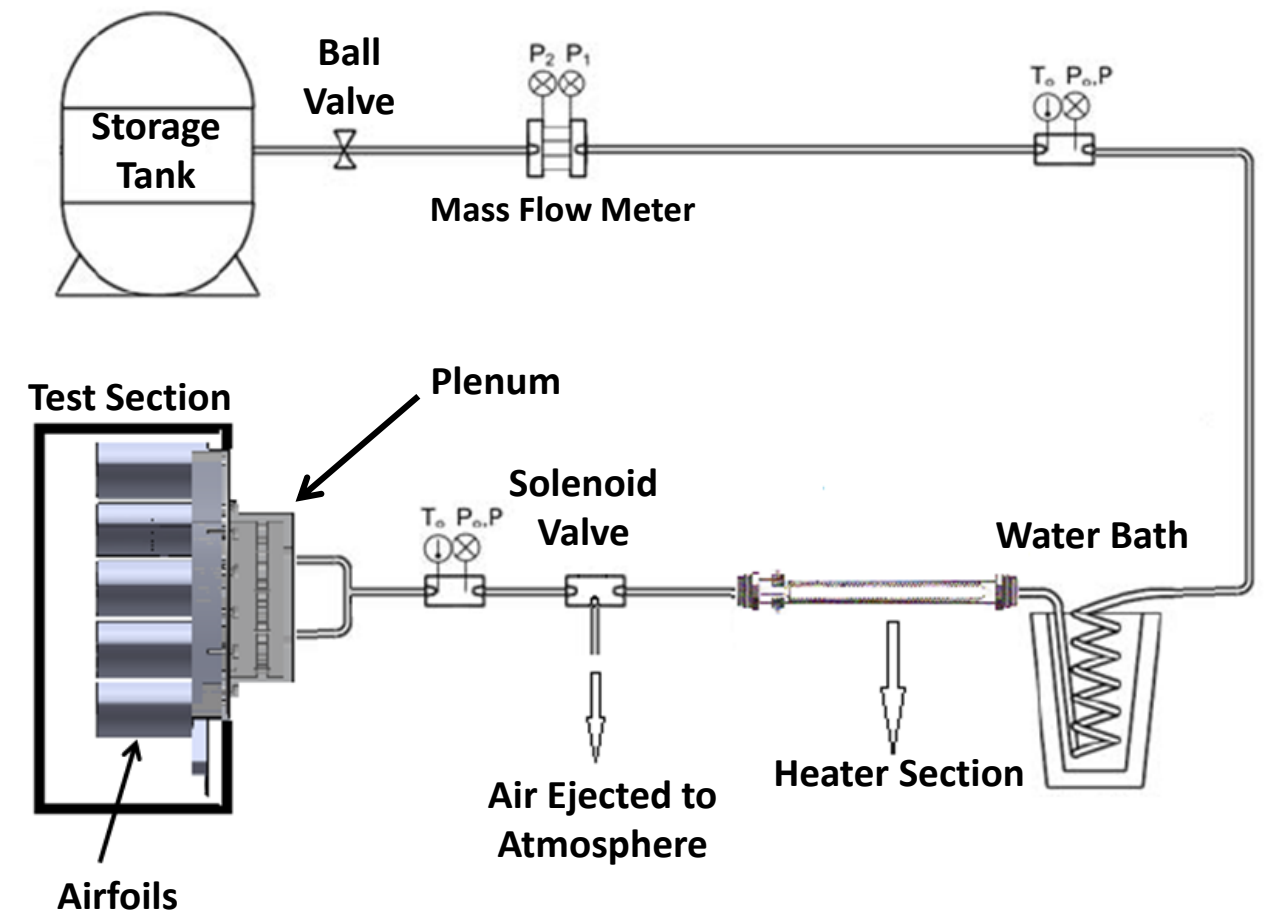

Figure 8 : Coolant System

Figure 9 shows a closer look at the location of the coolant plenum on the airfoils. It has been established in past studies that the coolant does not impact losses as much as the presence of the slot itself. This was seen for upstream slot in Blot et al [22]. The focus of this study, is the geometric presence of the mateface gap itself. Therefore, coolant was only injected using the upstream slot and the value was maintained at $1 \%$ MFR for all three endwall geometries. 

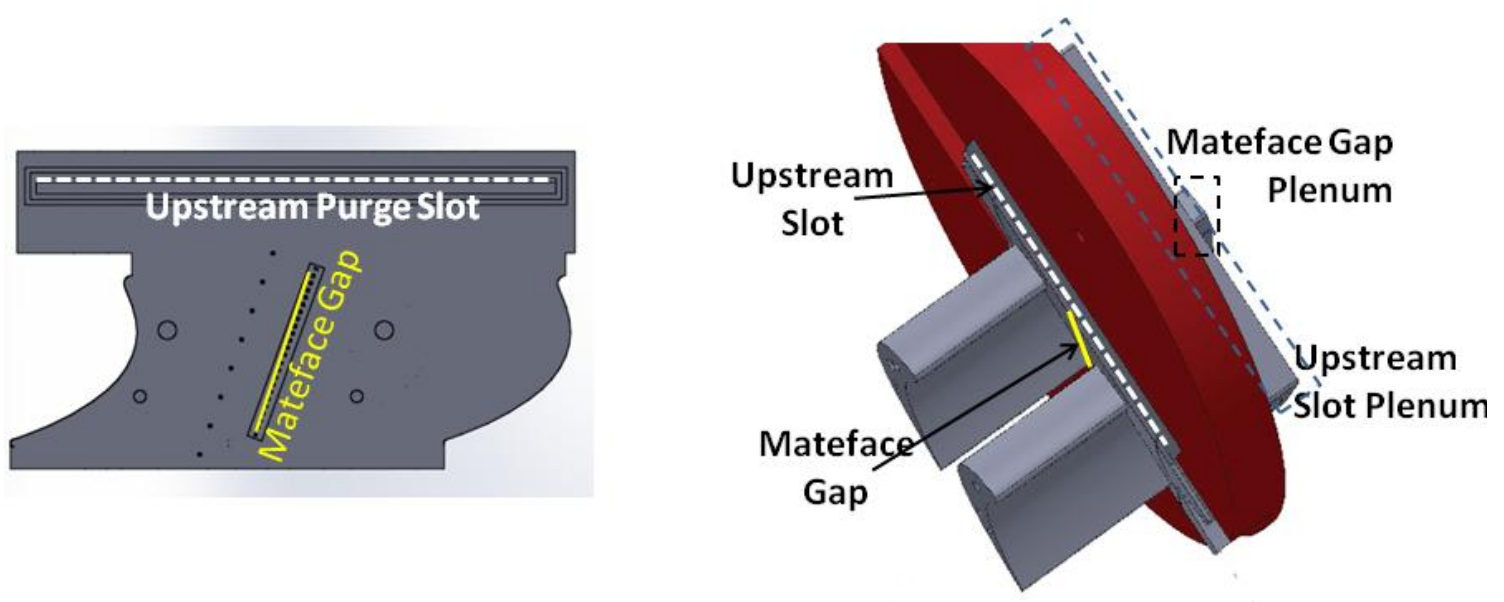

Figure 9 : Coolant Plenum Location

\subsection{Aerodynamic Measurement Technique}

\subsubsection{Upstream Measurements}

The test section had provision for upstream measurements by means of a slot provided 0.45 Cax upstream of the cascade. This was utilized for turbulence intensity measurements, which was set at $8 \%$ by means of a turbulence grid placed $5.5-\mathrm{C}_{\mathrm{ax}}$ upstream of the cascade, shown in Figure 7. The upstream pitchwise traverse measurements using a Pitot probe established the flow uniformity deviation within \pm 0.4 and also provided the upstream boundary layer thickness. [9] The same location was utilized for introducing a pitot probe for measuring the incoming total pressure and the static pressure which were used in calculating the incoming Mach number, and also for calculating the loss coefficient for comparing aerodynamic performance.

\subsubsection{Downstream Measurements}

For this study, the measurements were made at 1.0 Cax. The losses tend to be mixed out and the individual flow features are difficult to distinguish. The periodicity of flow does 
deteriorate somewhat since the flow starts turning towards the exit of the cascade. However, the location was chosen since it yields a better estimate of reduction of losses due to endwall contouring, as reported by Abraham et al [21] where they saw a $3 \%$ reduction based on the measurements at 0.1 Cax which magnified to $17 \%$ at $1.0 \mathrm{Cax}$. This gives a more definitive picture beyond the uncertainty level of the measurements. While the contours themselves may not be very reliable indicators in pinpointing specific flow features on the endwall, the results can offer insight with regards to a general trend with respect to the relative impact with variation geometry, cooling features etc and have been discussed in this study with that consideration. For static pressure measurement, $1.6 \mathrm{~mm}$ static pressure taps were placed $0.5-C_{a x}$ downstream of the airfoil trailing edge.

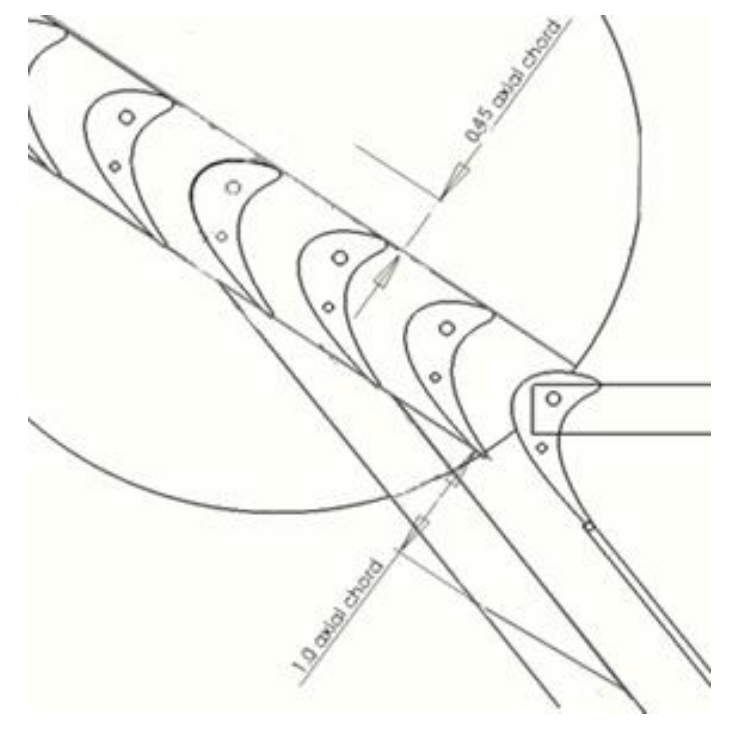

Figure 10 : Pressure Probe locations

The 5-hole probe used was provided by Aeroprobe Corp. It was mounted in a traverse arrangement 1.0 Cax downstream. The arrangement utilized a carriage assembly to hold the probe securely, as it was driven through the pitch by a stepping motor(Figure 11). 


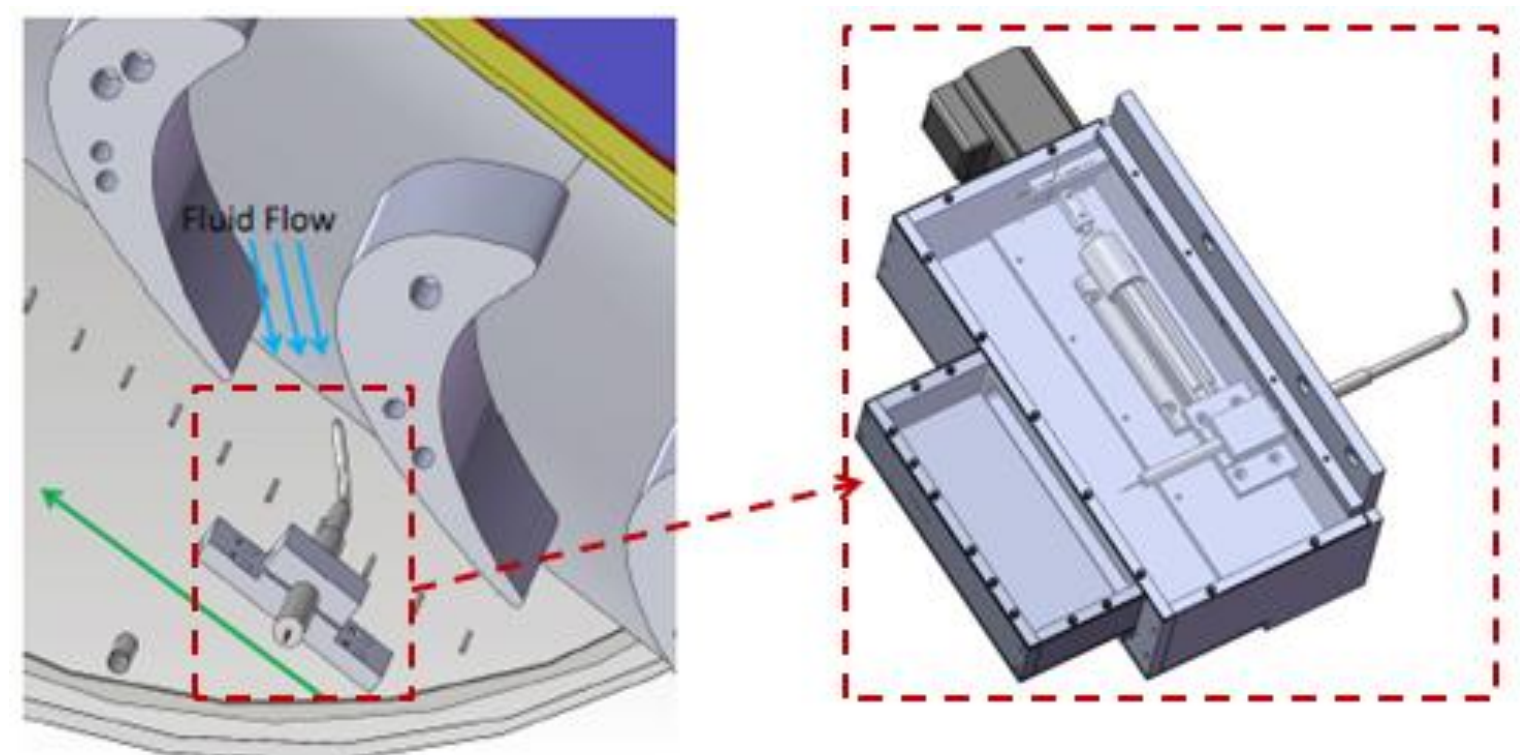

Figure 11 :Traverse Arrangement

Figure 12 gives a detailed visual of the data acquisition carried out by the traverse. The sampling window was for 6 seconds, positioned in the steady region of the tunnel run. This resulted in 61 data points, and it covered a little over the passage length beyond both trailing edges for the passage of interest. Also, between subsequent runs the probe was moved along the span locations, in smaller increments near the endwall and larger increments as it approached midspan. This yielded about 15 data points along the spanwise direction covering about $60 \%$ of the span. This helped in obtaining a grid of data points which was sufficiently resolved to chart the flow characteristic 1.0 Cax downstream. The usable data however was considered only until half span, and the data beyond the trailing edges was not considered. The first set of data points extremely close to the wall which was taken as "0" during measurement were also discarded for consistency between different cases, since the exact probe distance from the endwall was not possible to measure. Pressure measurements were collected using the Netscanner 94RK pressure scanner. 


\begin{tabular}{|l|l|l|}
\hline \multicolumn{1}{|c|}{ Direction } & Measurement & $\begin{array}{c}\text { Usable } \\
\text { data for } \\
\text { half span }\end{array}$ \\
\hline $\begin{array}{l}\text { Pitchwise } \\
\text { (5HP movement } \\
\text { through stepping } \\
\text { motor) }\end{array}$ & $\begin{array}{l}6 "=6 \text { s run time } \\
=1.34 \text { pitch length }\end{array}$ & $\begin{array}{l}\text { One pitch } \\
\text { (TE to TE) }\end{array}$ \\
\hline $\begin{array}{l}\text { Spanwise } \\
\text { (5hp moved } \\
\text { manually between } \\
\text { runs) }\end{array}$ & $\begin{array}{l}4.025 " \\
=\sim 67 \% \text { span }\end{array}$ & Half Span \\
\hline \begin{tabular}{l} 
Sufficiently resolved grid for half passage \\
\hline
\end{tabular}
\end{tabular}

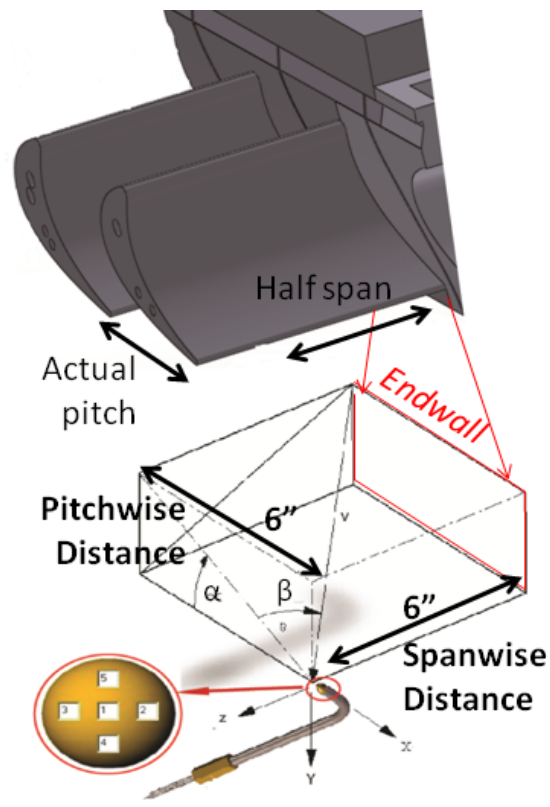

Figure 12 : Setup for traverse data acquisition

Blade loading measurements without the upstream slot were carried out by Abraham et al. [21]. The center airfoil was instrumented to calculate static pressure to obtain the isentropic Mach number, which showed good agreement with the CFD.

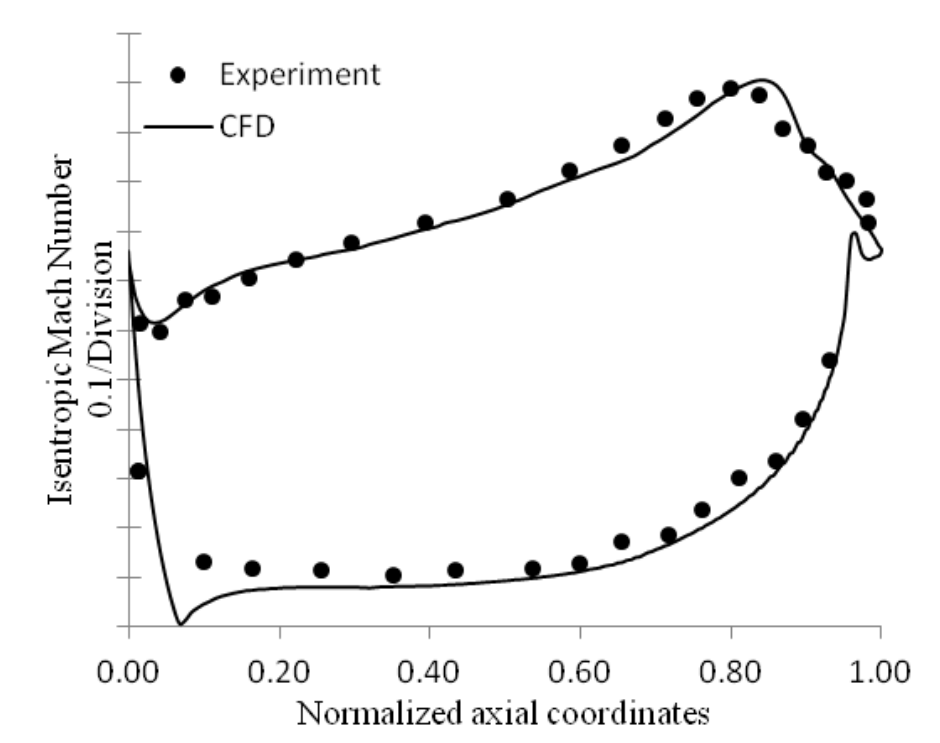

Figure 13 :Midspan airfoil loading from CFD and experiments [21] (Figure 5 Effect of Endwall Contouring on a Transonic Turbine Blade Passage:Part 1 - Aerodynamic Performance", ASME Paper No. GT2012-68425. Used under fair use, 2013) 


\subsection{Data Reduction \& Loss Coefficient Calculation}

\subsubsection{Formulation}

The aerodynamic performance was compared based on pressure loss at $1.0 \mathrm{Cax}$ downstream and multiple pitch and span points were covered to generate a picture of the flow conditions through the passage. The loss coefficients for test runs with and without blowing cases were measured for both the planar and contoured endwall. For the planar case, the performance was compared to the geometry with only the slot, as was reported by Blot et al. [22].

A standard 5-hole probe data reduction algorithm is utilized based on the pitch and yaw coefficients, in conjunction with the calibration database (supplied by Aeroprobe along with the 5 hole probe), to calculate total exit pressure and velocity components from the five measured pressures. Utilizing the equations below the code uses a hyper-surface fitting for the multidimensional Mach number calibration data base in order to interpolate between coefficients developed from the calibration data and the experimental data.

$$
\begin{array}{ll}
\overline{\mathrm{P}}=\frac{\mathrm{P}_{2}+\mathrm{P}_{3}+\mathrm{P}_{4}+\mathrm{P}_{5}}{4} & \text { Equation [2] } \\
C p_{\text {yaw }}=\frac{\left(P_{2}-P_{3}\right)}{\left(P_{1}-\bar{P}\right)} & \text { Equation [3] } \\
C p_{\text {pitch }}=\frac{\left(P_{4}-P_{5}\right)}{\left(P_{1}-\bar{P}\right)} & \text { Equation [4] } \\
C p_{M a}=\frac{\left(P_{1}-\bar{P}\right)}{\left(P_{1}\right)} & \text { Equation [5] }
\end{array}
$$


The values for individual data points utilize the difference in the inlet and exit total pressure, and the normalizing factor used throughout is the difference between mass averaged total exit pressure and area averaged static pressure for the test grid.

$$
\boldsymbol{\omega}=\frac{P t_{i n}-P t_{e x}}{P t_{e x(m)}-P s t_{e x(a)}} \quad \text { Equation [6] }
$$

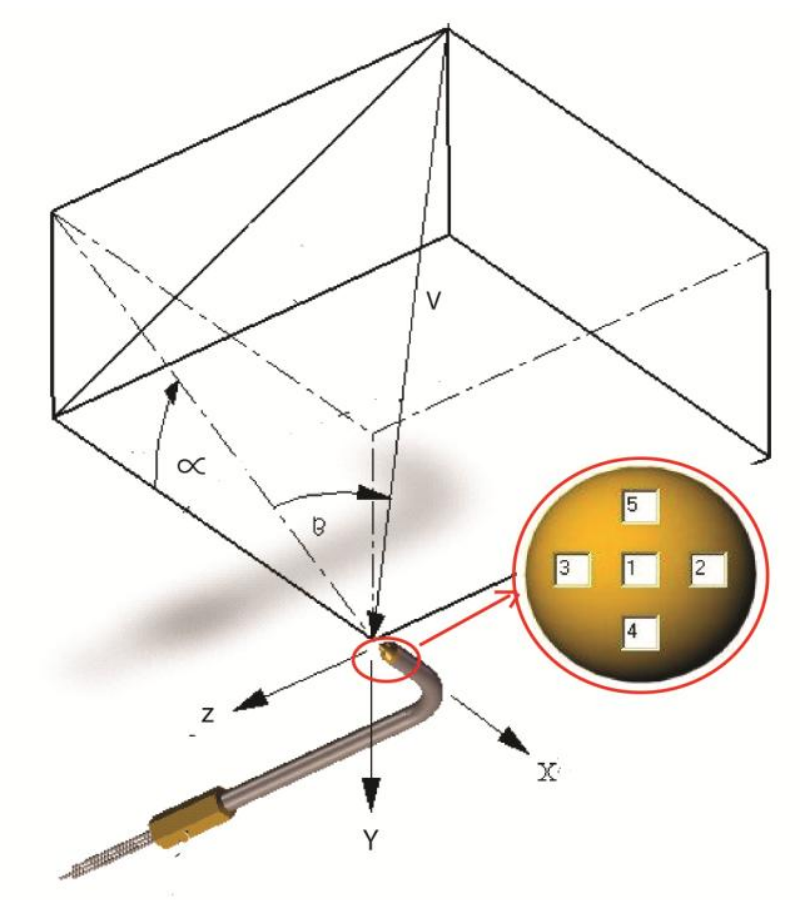

Figure 14 : 5- Hole Probe Orientation

For comparing the overall performance of a particular configuration, a composite loss value has been calculated. By definition, it is the difference between the mass averaged exit total pressure and the averaged inlet total pressure (accounting for the boundary layer effect for which the correction is incorporated, represented in formula as $P t_{\text {in(average) }}$ ), and this difference is normalized by the difference between mass averaged exit total pressure and area averaged exit static pressure for the test grid. 


$$
\omega_{\text {overall }}=\frac{P t_{\text {in (average })}-P t_{\text {ex }(m)}}{P t_{\text {ex }(m)}-P s t_{e x(a)}}
$$

Equation [7]

These could be computed using the total temperature measure upstream of the cascade, and the exit mach number, static pressure and total pressure derived from the five hole probe.

\subsection{Uncertainty in Loss Coefficient Measurements}

The average uncertainty in the calculation of $\omega$ values (loss coefficient) was calculated using Kline and McClintok's method [24].The uncertainty level follows a non linear relation with the loss coefficient value. For the range of loss coefficients encountered in this study, the uncertainty percentage varies from 3-8\%.

Applying Kline and McClintock's [24] method

$$
\begin{gathered}
w_{\omega}=\left[\left(\frac{\partial \omega}{\partial p_{t_{-} i n}} w_{p t_{-} i n}\right)^{2}+\left(\frac{\partial \omega}{\partial p_{t_{-} e x}} w_{p t_{-} e x}\right)^{2}+\left(\frac{\partial \omega}{\partial p_{s_{-} e x}} w_{p s_{-} e x}\right)^{2}\right]^{\frac{1}{2}} \\
w_{\omega}=\left[\left(\frac{\partial \omega}{\partial p_{t_{-} i n}} w_{p t_{-} \text {in }}\right)^{2}+\left(\frac{\partial \omega}{\partial p_{t_{-} e x}} w_{p t_{-} e x}\right)^{2}+\left(\frac{\partial \omega}{\partial p_{s_{-} e x}} w_{p s_{-} e x}\right)^{2}\right]^{\frac{1}{2}} \quad \text { Equation [8] }
\end{gathered}
$$

Where, $w=$ uncertainty

$$
\begin{aligned}
\frac{\partial \omega}{\partial p_{t_{\_} i n}} & =\frac{1}{\left(p_{t_{-} e x}-p_{s_{-} e x}\right)} & & \text { Equation [9] } \\
\frac{\partial \omega}{\partial p_{t_{\_} e x}} & =-\frac{(1+\omega)}{\left(p_{t_{\_} e x}-p_{s_{-} e x}\right)} & & \text { Equation [10] } \\
\frac{\partial \omega}{\partial p_{s_{-} e x}} & =\frac{\omega}{\left(p_{t_{-} e x}-p_{s_{-} e x}\right)} & & \text { Equation [11] }
\end{aligned}
$$

Combining equation [2-5] yields, 


$$
\begin{gathered}
w_{\omega}=\left[\left(\frac{1}{\left(p_{t_{-} e x}-p_{s_{-} e x}\right)} w_{p t_{-} i n}\right)^{2}+\left(-\frac{(1+\omega)}{\left(p_{t_{-} e x}-p_{s_{-} e x}\right)} w_{p t_{-} e x}\right)^{2}+\left(\frac{\omega}{\left(p_{t_{-} e x}-p_{s_{-} e x}\right)} w_{p s_{-} e x}\right)^{2}\right]^{\frac{1}{2}} \\
\Rightarrow \frac{w_{\omega}}{\omega}=\frac{1}{\left(p_{t_{-} e x}-p_{s_{-} e x}\right)}\left[\left(\left(1+\frac{1}{\omega}\right) w_{p t_{-} e x}\right)^{2}+\left(\frac{w_{p t_{\text {in }}}}{\omega}\right)^{2}+\left(w_{p s_{-} e x}\right)^{2}\right]^{\frac{1}{2}} \quad \text { Equation [12] }
\end{gathered}
$$

The isentropic Mach number definition is defined on $p_{t_{-} i n}$ and $p_{s_{-} e x}$. Assuming the process is isentropic, it can be stated that $p_{t_{-} e x}=p_{t_{-} i n}$. After substituting, $\frac{p_{t_{-} i n}}{p_{s_{-} e x}}=\left(1+\frac{\gamma-1}{2} M_{i s o}^{2}\right)^{\frac{\gamma}{\gamma-1}}$ into equation (6) gives

$$
\frac{w_{\omega}}{\omega}=\frac{1}{p_{s_{-} e x}\left[\left(1+\frac{\gamma-1}{2} M_{i s o}^{2}\right)^{\frac{\gamma}{\gamma-1}-1}\right]}\left[\left(\left(1+\frac{1}{\omega}\right) w_{p t_{-} e x}\right)^{2}+\left(\frac{w_{p t_{i n}}}{\omega}\right)^{2}+\left(w_{p s_{-} e x}\right)^{2}\right]^{\frac{1}{2}} \quad \text { Equation [13] }
$$

Now for a given Mach number and related $p_{s_{-} e x}$, we can calculate uncertainty in experimental results for a given value of loss coefficient, if the uncertainties in individual variables are known.

a) Uncertainty in $\boldsymbol{p}_{\boldsymbol{t}_{-} i \boldsymbol{i}}$ : The total pressure $\left(p_{t_{-} i n}\right)$ is measured using $9816-2675$ module with a 20 psi range. Instrument accuracy is $0.05 \% \mathrm{FS}$, which is $\pm 0.01 \mathrm{psi}$. Also, over the window of data collection, the maximum variation in the recorded pressure value is \pm 0.05 psi. Hence, the total uncertainty (Instrument accuracy + uncertainty due to unsteadiness of total pressure) can be given as,

$$
w_{p t_{-} i n}= \pm 0.06 p s i
$$

b) Uncertainty in $\boldsymbol{p}_{\boldsymbol{t}_{-} \boldsymbol{e x}}$ : The instrument accuracy remains the same since the same equipment is being used. This pressure is being recorded by means of a traversing 5-HP 
probe and each port may have its own uncertainty value which would be difficult to combine. For a close estimation, the final pressure value obtained from data reduction for two repeatability cases were compared. A difference of about 0.16 psi was found in pressure values for respective traverse location, and hence this gives an uncertainty of \pm 0.08 psi . Combining with instrumental uncertainty, we get

$$
w_{p t_{-} e x}= \pm 0.09 p s i
$$

c) Uncertainty in $\boldsymbol{p}_{s_{-} e x}: \boldsymbol{p}_{s_{-} e x}$ is the wall static pressure measured 0.5 axial chord downstream. The values are generally below $2.5 \mathrm{psi}$ and the instrument accuracy is 0.15 $\%$ FS, i.e. $\pm 0.03 \mathrm{psi}$. The variation in static pressure between two repeatability runs for points at respective sampling times is a maximum of $\pm 0.003 \mathrm{psi}$. Hence it is seen here that the instrumental uncertainty dominates. Combining the two, we get,

$$
w_{p s \_x}= \pm 0.033 \text { psi }(10)
$$

Hence, uncertainty in all the variables is known and can be substituted in eqn 7 . The equation (7) can hence be simplified to yield,

$$
\frac{w_{\omega}}{\omega}=\frac{1}{14.6\left[\left(1+\left[\frac{1.4-1}{2}\right] * 0.87^{2}\right)^{\frac{1.4}{1.4-1}}-1\right]}\left[\left(\left(1+\frac{1}{\omega}\right)( \pm 0.06)\right)^{2}+\left(\frac{( \pm 0.09)}{\omega}\right)^{2}+\right.
$$

$$
( \pm 0.033) 212 \quad \text { Equation [14] }
$$

The correlation listed above can be used to plot the expected uncertainty against the absolute value of loss coefficient obtained. It can be observed that percent uncertainty is inversely proportional to the loss coefficient value. At an uncertainty of $8 \% \omega=0.141$ giving 
a $\Delta \omega= \pm 0.0112$, and at about $2.8 \%$ uncertainty at $\omega=0.373$ results in $\Delta \omega= \pm 0.0107$. Conservatively we can say

$$
\boldsymbol{\omega}_{\text {actual }}=\boldsymbol{\omega}_{\text {measured }} \pm \mathbf{0 . 0 1 1 2} \quad \text { Equation [15] }
$$

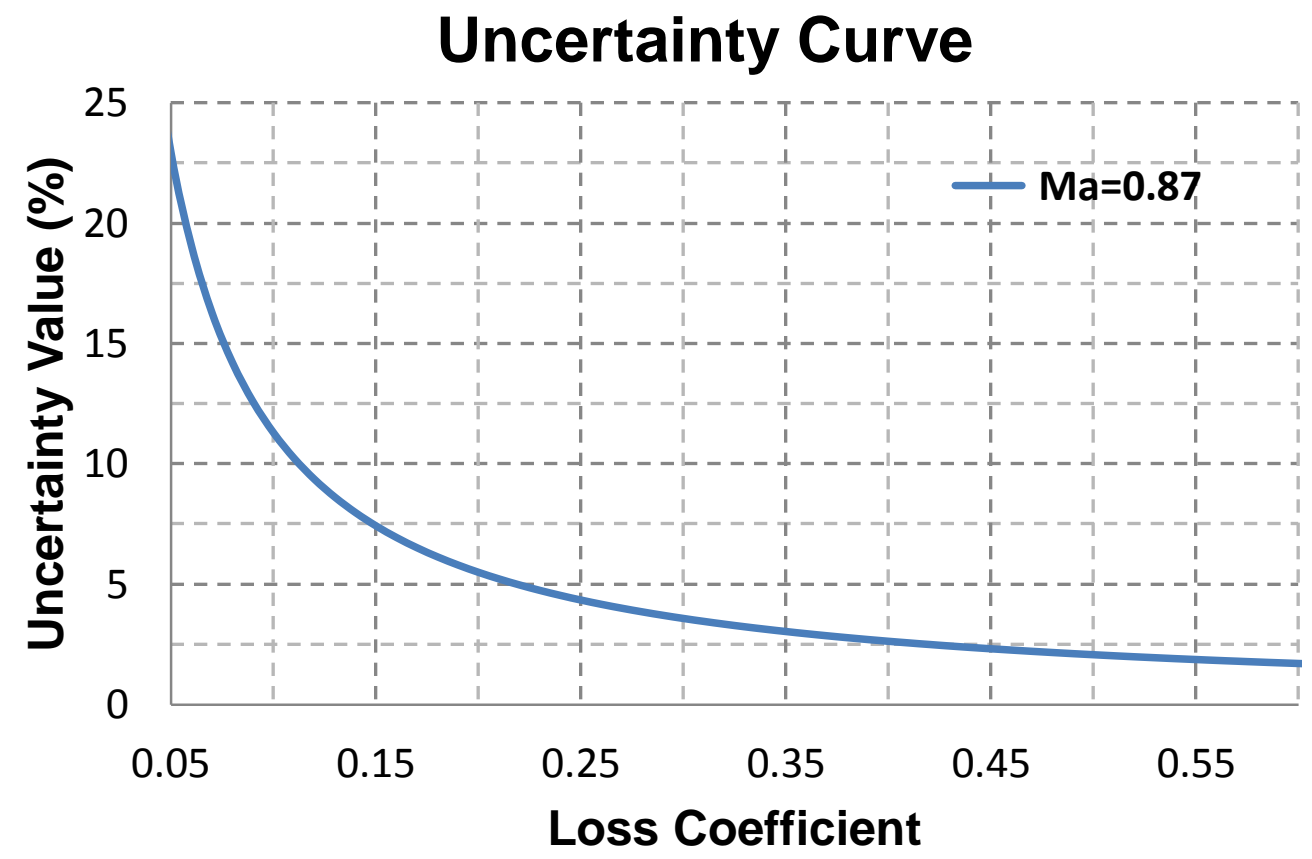

Figure 15 : Uncertainty Correlation with loss Coefficient for $\mathrm{Ma}=0.87$ 


\section{Chapter 4 : Results and Discussion}

\subsection{Overview}

Figure 16 presents the parametric effects that were sought to be addressed in this study. These have been arranged in the order of their importance on the impact they have on the losses. The geometries available for making these comparisons have been listed on the right in Figure 16 .
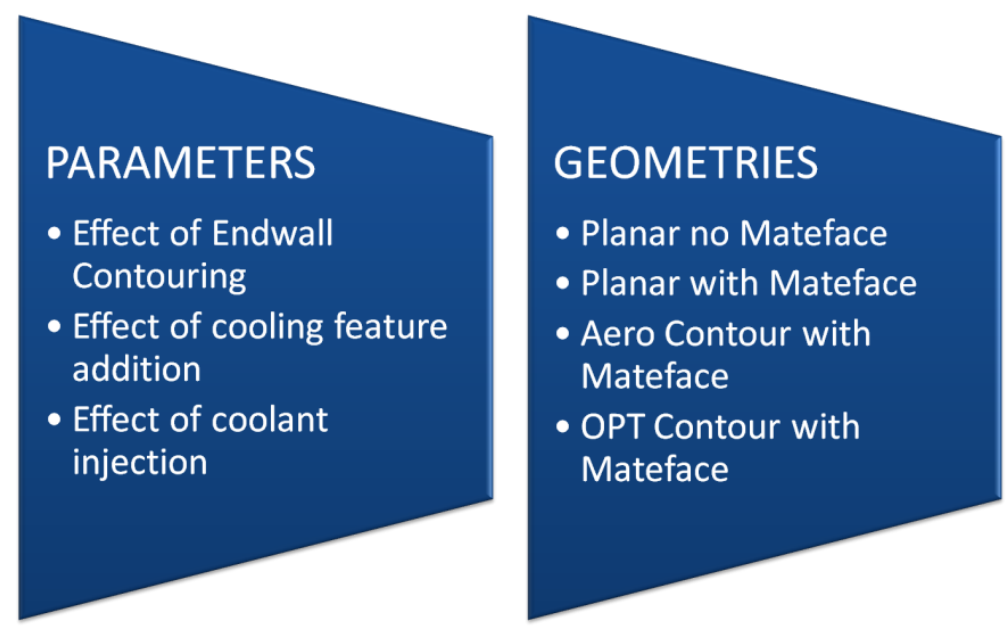

Figure 16 : Parameters for Aerodynamic Performance Study

The effect of presence of the mateface gap as a geometric disruption has been examined and compared with a case having no mateface gap. The effect of coolant addition through the upstream purge slot has also been examined for both endwalls. The final comparison would be between the planar and the contoured endwall. Table 1 lists the test cases that have been performed on both endwalls. As mentioned earlier, the losses are highly mixed out, and no definite flow features are apparent. However, the pitchwise averaged plots of loss coefficient along the span indicate a trend, which shall be explained in light of the impact of endwall interactions on secondary flow. 


\begin{tabular}{|c|c|c|c|c|c|}
\hline \multirow[t]{2}{*}{ CASE } & \multicolumn{2}{|c|}{ Contouring } & \multirow{2}{*}{$\begin{array}{l}\text { Upstream } \\
\text { Slot }\end{array}$} & \multirow{2}{*}{$\begin{array}{l}\text { Mateface } \\
\text { Gap }\end{array}$} & \multirow{2}{*}{$\begin{array}{c}\text { Coolant } \\
\text { (Location,\% MFR) }\end{array}$} \\
\hline & Aero & OPT & & & \\
\hline P0 & \multicolumn{2}{|c|}{$x$} & $\checkmark$ & $x$ & Slot $0 \%$ \\
\hline P1 & \multicolumn{2}{|c|}{$x$} & $\checkmark$ & $\checkmark$ & Mateface $0 \%$, Slot $0 \%$ \\
\hline P2 & \multicolumn{2}{|c|}{$x$} & $\checkmark$ & $\checkmark$ & Mateface $0 \%$, Slot $1 \%$ \\
\hline A1 & $\checkmark$ & & $\checkmark$ & $\checkmark$ & Mateface $0 \%$, slot $0 \%$ \\
\hline A2 & $\checkmark$ & & $\checkmark$ & $\checkmark$ & Mateface $0 \%$, slot $1 \%$ \\
\hline 01 & & $\checkmark$ & $\checkmark$ & $\checkmark$ & Mateface $0 \%$, slot $0 \%$ \\
\hline 02 & & $\checkmark$ & $\checkmark$ & $\checkmark$ & Mateface $0 \%$, slot $1 \%$ \\
\hline
\end{tabular}

Table 1 : Test Matrix

While pitchwise averages may not be adequate in recognizing differences in the flow features, a better picture emerges on plotting loss contours for the span area covered. The loss coefficient calculated at every data point has been consolidated in the form of contour plot. This helps in visualizing areas with similar pressure loss which can then be used to predict the physical flow phenomenon. The loss contours have all been plotted using a common scale to present a better visual comparison basis. All loss contours have been labeled as $A B C D$, where in edge $A D$ represents the trailing edge for the top blade in the cascade, and $B C$ is the lower trailing edge. Points $C$ and $D$ lie on the midspan of the respective trailing edges. The relative placement of the contour plane $(A B C D)$ can be referred from Figure 30(a) which has been reproduced [8] and annotated for better clarity and this nomenclature shall be referred throughout the coming sections. 


\subsection{Oil Flow Visualization}

Oil flow visualization is a technique which can help to investigate surface flow features through streak lines. For further clarity in determining flow patterns in the presence of the Upstream slot, the mateface gap and a contoured endwall, two oil flow visualizations were performed. One was for the geometry case P0, and the other for the case $\mathrm{O} 1$. Fluorescent colored powders were mixed with high viscosity oil, which were then applied to the cascade geometry in situ. The endwall was painted green in both cases, but for P0, the pressure side of the airfoil was red and the suction side was painted in blue while the convention was reversed for $\mathrm{O} 1$.

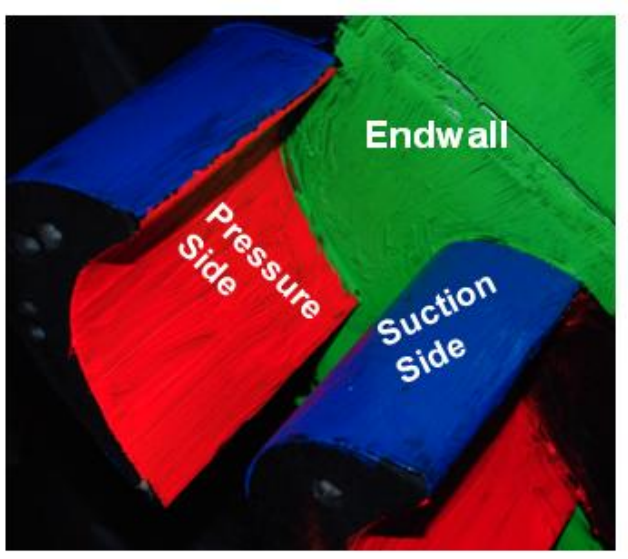

(a)

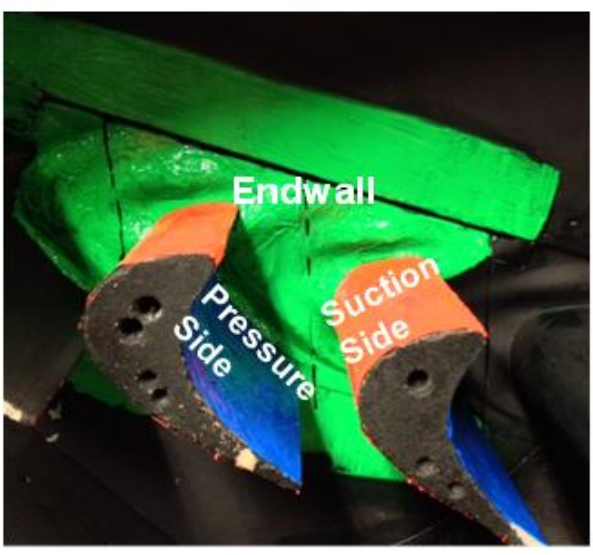

(b)

Figure 17 : Color scheme before running (a) P0 (b) 01 
a) Case P0

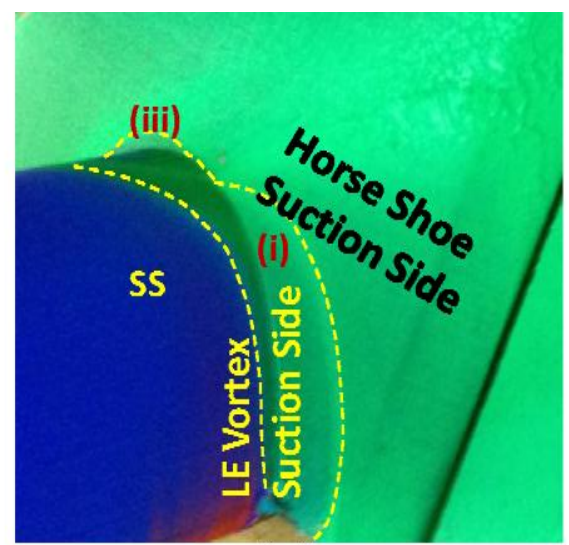

(a)

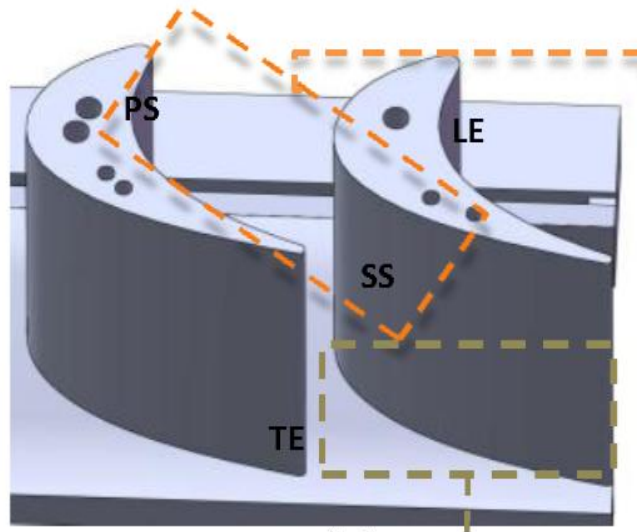

(c)
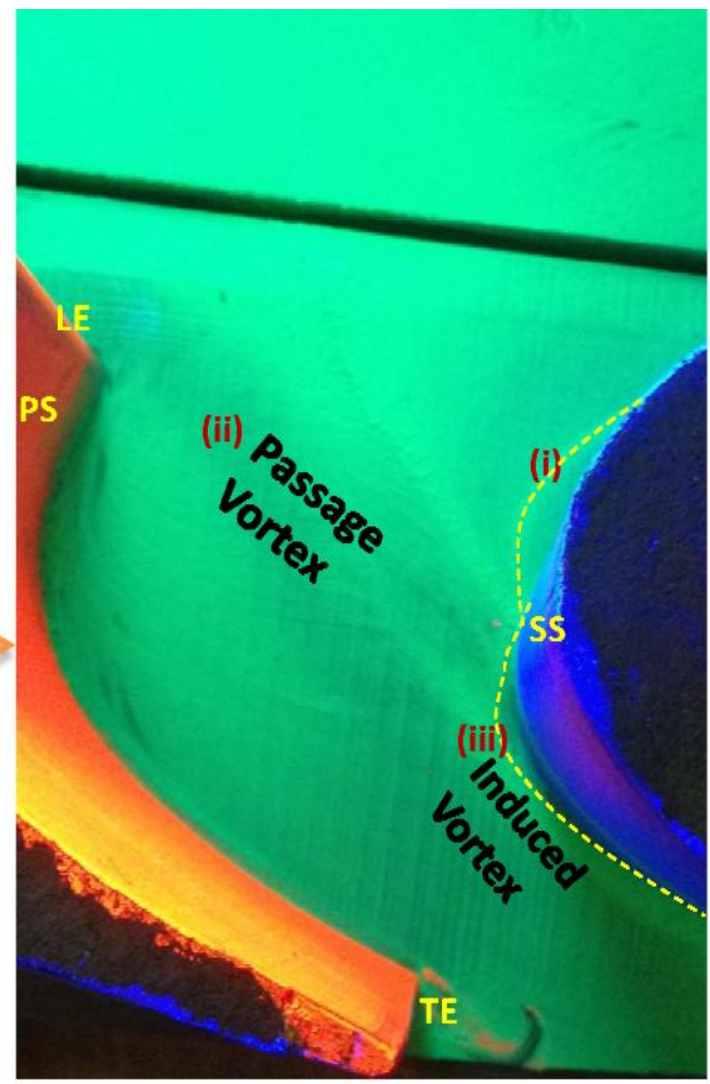

(b)

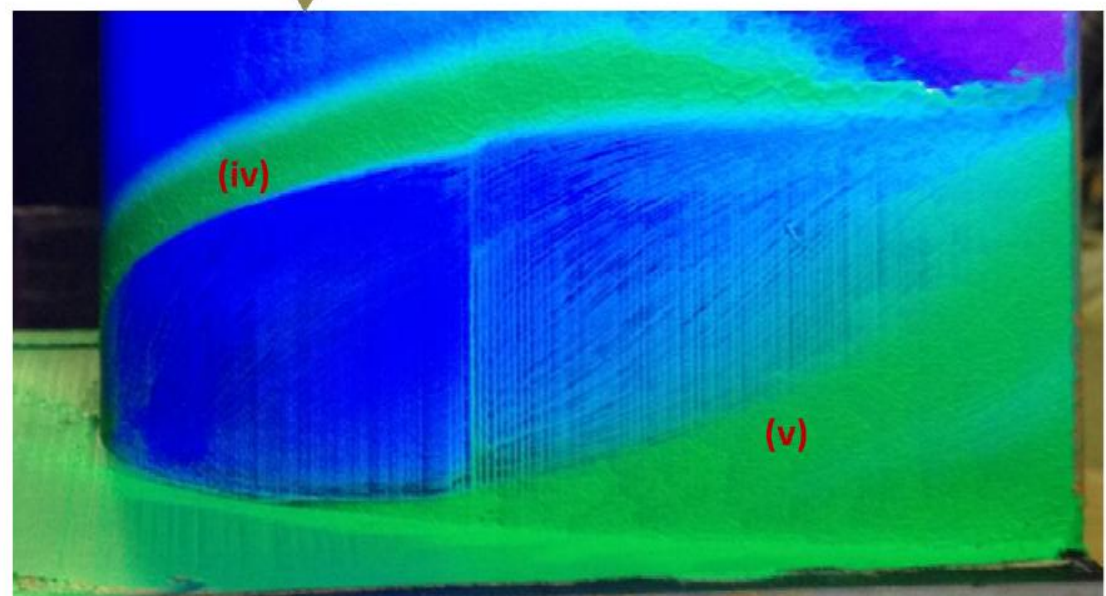

(d)

Figure 18 : Oil Flow Visualization Results for P0 
Figure 18 shows the results of oil flow visualization conducted on the P0 case of a planar endwall with only the upstream slot. In Figure 18 (b) the dashed yellow lines have been added to indicate the trajectory of the accumulation of paint. Figure 18 (a) shows a different view of the leading edge where the vortex at the suction side leading edge is clearer, induced by the bifurcation of the incoming boundary layer. The point where the passage vortex (indicated as (ii)) meets the suction side, another vortex seems to have originated which continues along the airfoil and the endwall. The view presented in Figure 18 (d) shows a trailing edge perspective. Here, the lift off of the green paint is clearly visible, and this might be caused by the induced vortex follows this trajectory above the passage vortex. The lift off by the passage vortex itself is also visible at Figure 18(v). The height of this liftoff can signify the intensity and strength of the vortex, and was measured to be around 2 inches, i.e one third of the blade span. The pressure side does not show any significant flow features, except that the streaks along the endwall show the flow being swept towards the suction side owing to the pressure differential existing in the passage, which is dominant near the endwall. 
b) Case 01

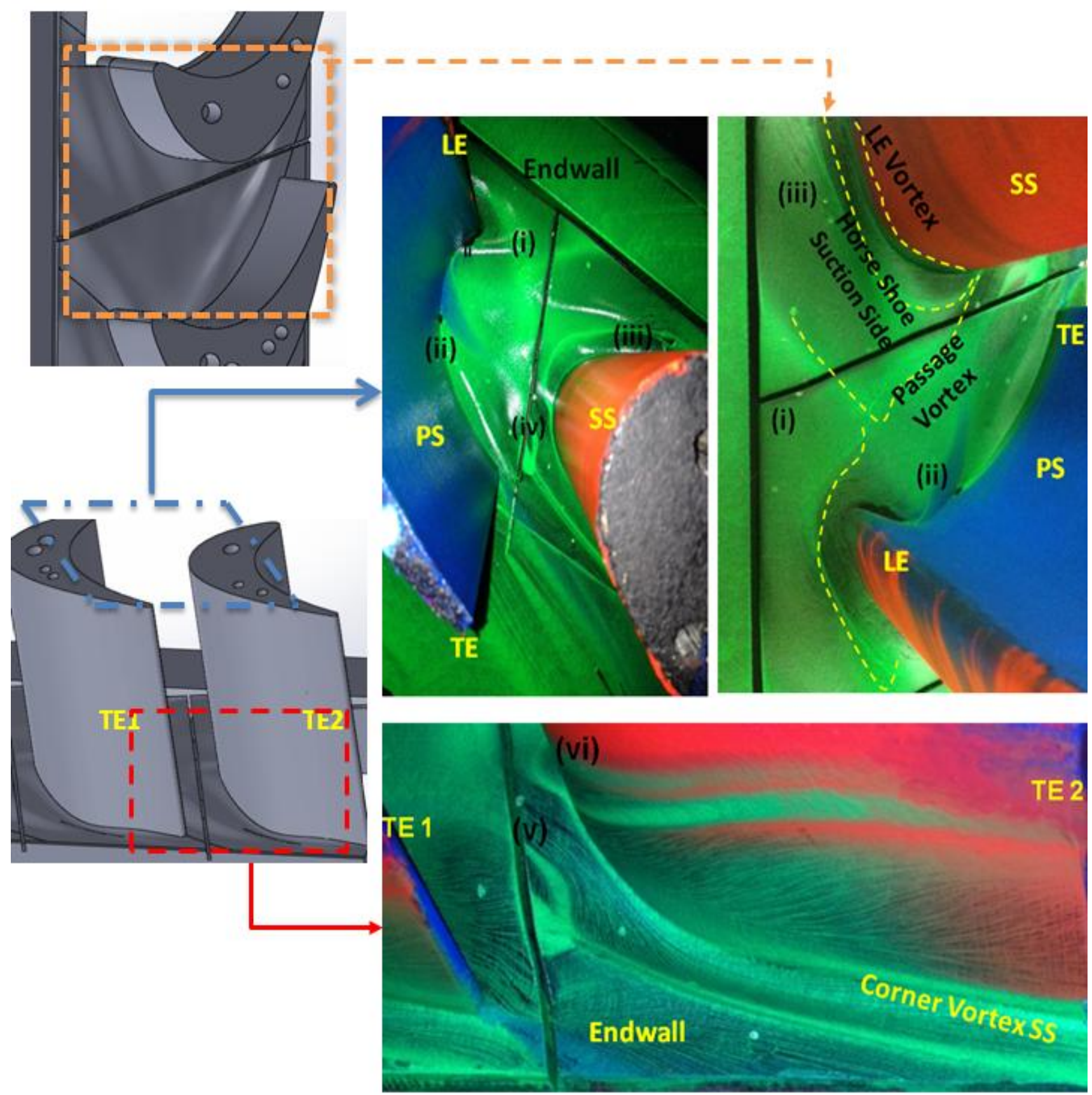

Figure 19 : Oil Flow Visualization for OPT Contour 
In Figure 19 we see the location of the saddle point which can be recognized from the accumulated paint at (i), and the bifurcation of the horseshoe vortex at the leading edge. At region (ii), the flow streamlines will be somewhat sparse, and the pressure leg of the horseshoe vortex as well as the leading edge vortex originate. The suction side leg of the horseshoe vortex travels along the suction side as seen in region (iii). At (iv), the effect of the pressure gradient on the endwall is quite clear as the blue paint from the suction side is drawn into the passage. The flow lines have been marked in the image in yellow dashed line. The passage vortex is visible in all three panels, and has been marked out in the right panel as it crosses over the mateface gap and meets the suction side. The view is cut off at point (e) which is the point of lift off for the passage vortex. It is continued from a different viewing angle in the bottom panel. The height to which it lifts off from the endwall has been indicated at the trailing edge. The leading edge suction side vortex is seen to roll up in the image on the right in Figure, and in the bottom panel it can be seen that it has lifted off along the suction side of the blade. It is formed by the flow originating from the inner boundary layer, thereby carrying the endwall green paint along with it. The corner vortex at the suction side is also present and adheres to the blade surface. These features, and their complex interaction eventually determine the aerodynamic performance due to secondary flow.

c) Comparison between $\mathrm{P} 0$ and $\mathrm{O} 1$

Figure 20 shows the differences between two geometries with respect to flow differences in the passage. Case $\mathrm{O} 1$ shows a clear saddle point, and some degree of separation near leading edge on the pressure side. The passage vortex is also delayed in O1, and this maybe attributed to both the mateface gap and the contouring which have weakened the passage crossflow. The contour guides the flow somewhat along the pressure side and it is pulled off in the passage only later, as seen at (iv) in Figure 21 (b). 


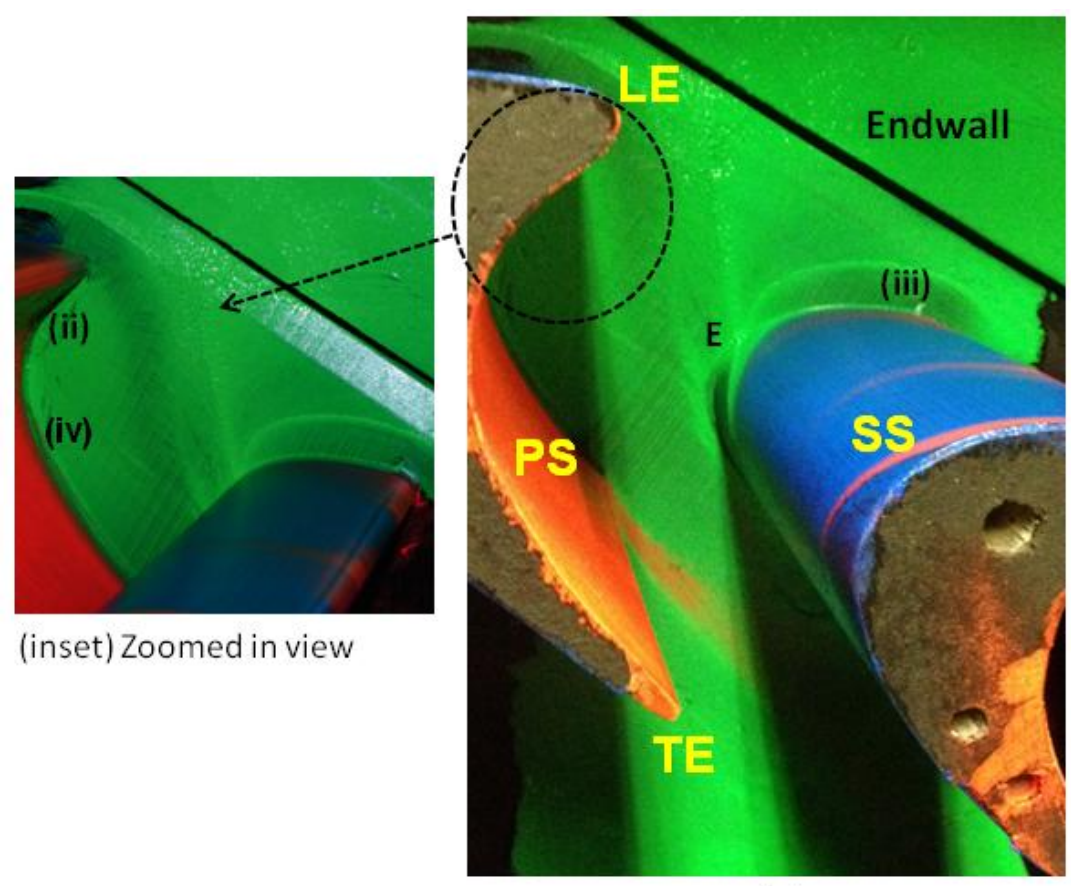

(a)

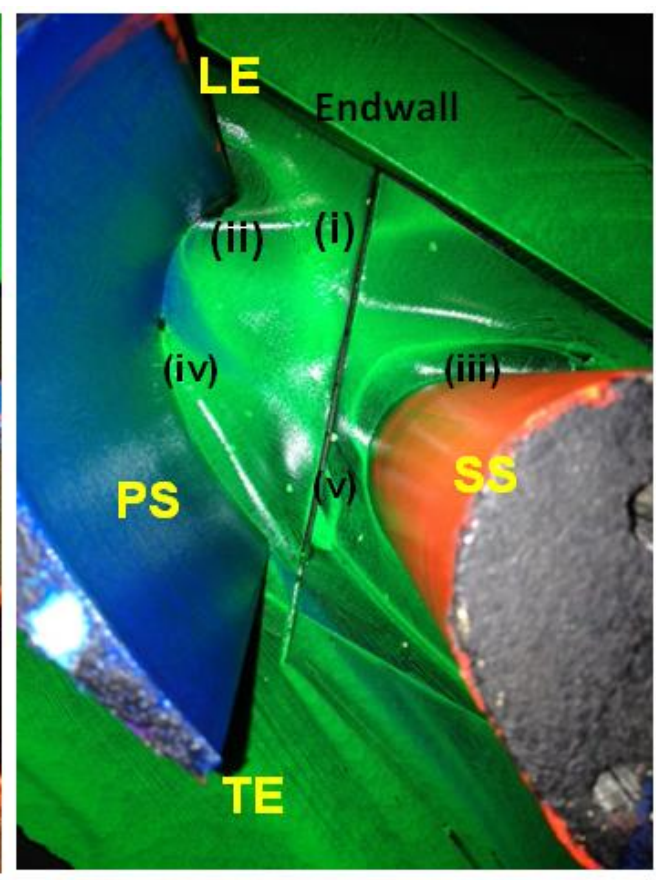

(b)

Figure 20 : Comparison between oil flow passage view (a) Case P0 (b) Case 01

In Figure 21, the trailing edge perspective for both $\mathrm{P} 0$ and $\mathrm{O} 1$ has been shown. As it can be seen that the $\mathrm{O} 1$ case has two induced vortices, and the passage vortex liftoff is less distinct as compared to P0 case. The height of lift off is also lesser. These translate to lower overall losses for the $\mathrm{O} 1$ case, as will be seen in following sections.

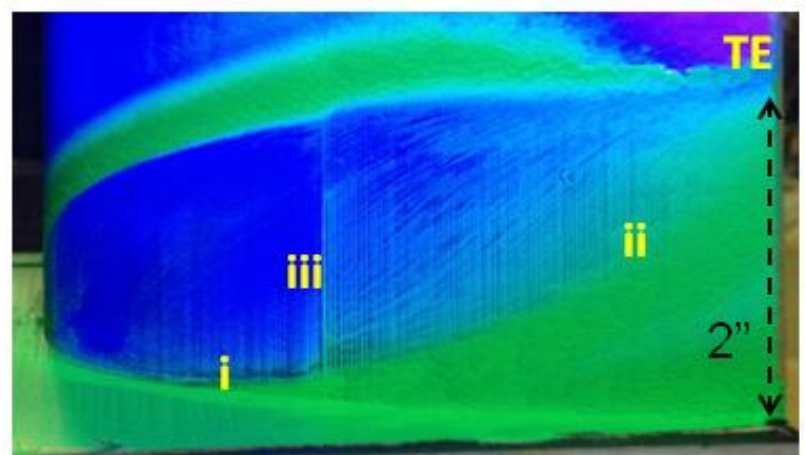

(a)

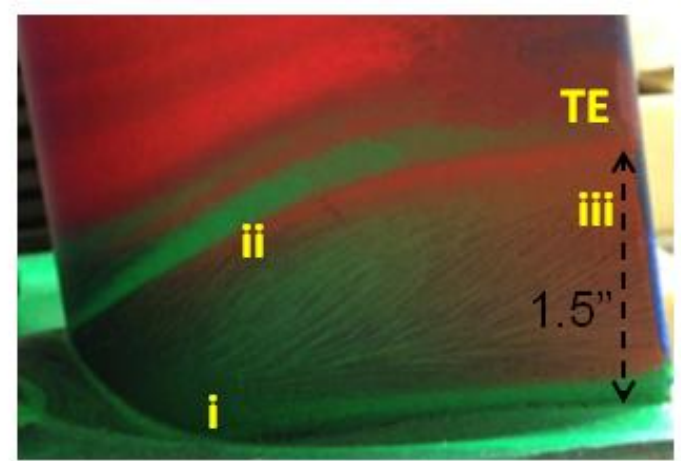

(b)

Figure 21 : Comparison between oil flow TE view (a) Case P0 (b) Case 01 


\subsection{Effect of Mateface Gap}

The mateface gap (also referred to in the literature as midpassage gap/slashface gap) represents the assembly gap between subsequent airfoils. There have been previous studies, as covered in the literature review section which explore the impact of the mateface gap on both aerodynamic and heat transfer performance. All these studies were conducted at low Mach numbers, and it was seen that the mateface gap contributes to mixed out losses. However, as Reid et al[19] observed, the presence of the mateface itself is the major cause and the losses increase only somewhat with increased flow rates. To ascertain the effect of the gap geometry, the losses for planar endwall with upstream slot were compared with losses for the planar endwall geometry with upstream slot as well as the mateface gap i.e, Case P0 with Case P1.

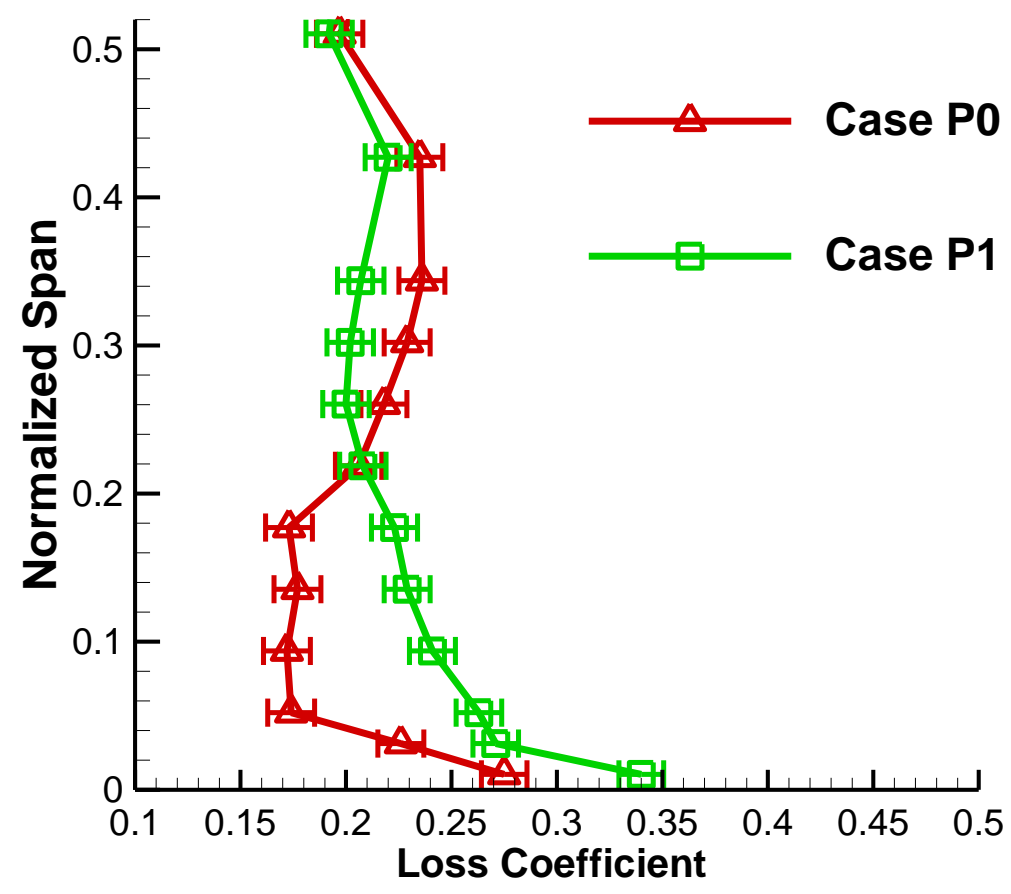

Figure 22 : Effect of Addition of Mateface with No Coolant Injection- Pitchwise Averaged Loss Coefficient 
On comparing pitchwise averaged loss coefficients for the half span Figure 22, it was seen that the addition of the mateface increased losses near the endwall up to $20 \%$ of the span and reduced them from $25-40 \%$ of the span. Since no coolant is being injected, it is expected that some mainstream flow might have been ingested in the mateface plenum. The mateface gap is expected to disrupt the crossflow by providing a discontinuity, and delay the progress of the passage vortex, thereby affecting overall secondary flow pattern. This can be visualized from Figure 30. Part (b) describes flow patterns near the endwall, which can be used to explain the loss contours generated using experimental data. On comparing P0 and P1from Figure 30(c) we see that the loss patterns across the passage have changed.

The case with mateface gap shows higher losses near the endwall, which can be explained by the possibility of ingestion in the mateface. The entrainment of low momentum flow in the mateface leaves lesser mass flow to be captured by the progressing passage vortex, which ,may explain the reduction in losses near midspan on corner ' $\mathrm{C}$ '(refer Figure 23). The low velocity flow adheres much more stronger to the endwall in the case where mateface gap is present. The discontinuity in the passage causes a disruption in the pressure gradient on the endwall. Thus passage vortex progresses slower and its intensity is decreased.

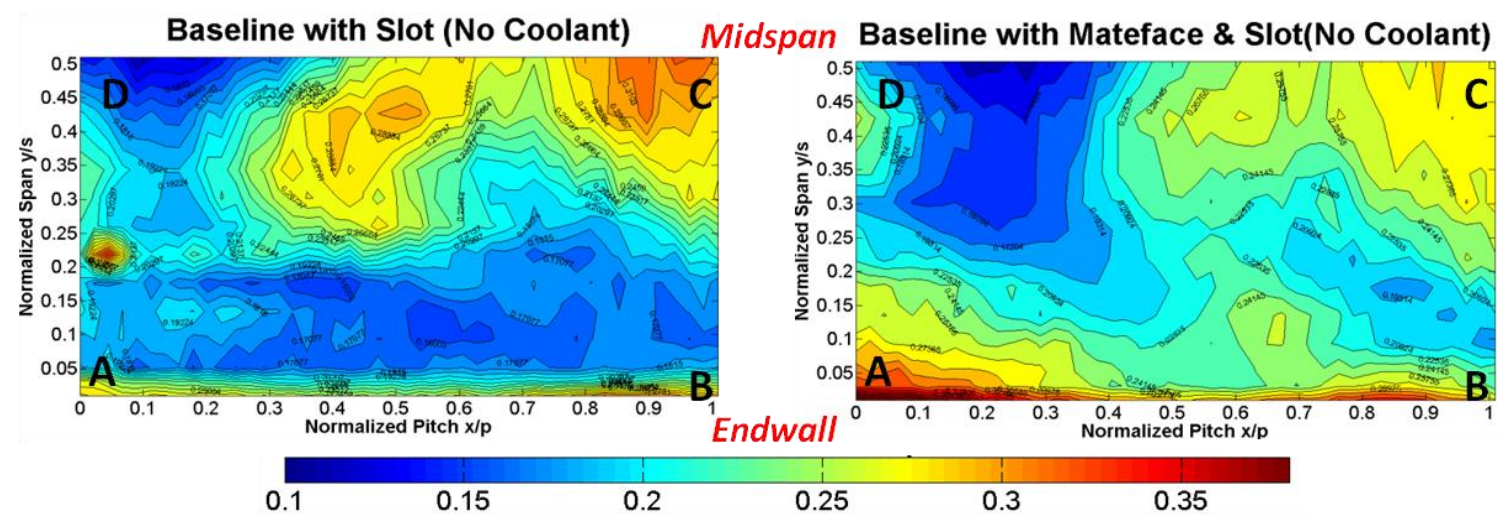

Figure 23 : Effect of Mateface Gap Addition - Loss Contours 


\subsection{Effect of coolant injection}

Coolant was injected only through the upstream slot, and it is seen from the pitch wise averaged plots in Figure 24 that the addition of coolant has some impact on the losses. For the planar wall case, the losses near endwall are more or less the same, and from $5-25 \%$ of the span the losses seem to have decreased due to the addition of coolant, after which the losses increase toward midspan. For the contoured cases, this effect is even more diminished in magnitude while trend of shift in losses appears to be similar to the planar endwall. Hence, for sake of explanation the case with maximum variation i.e. the planar endwall has been chosen for discussion. To get a more detailed insight into the possible reasons for change in loss distribution, the contour plots have been plotted in (Figure 25). These seem to suggest that the losses have been merely redistributed, and the change in secondary flow patterns might be primarily responsible for this change. Addition of coolant also causes higher mixing near endwall, and may lead to higher losses.

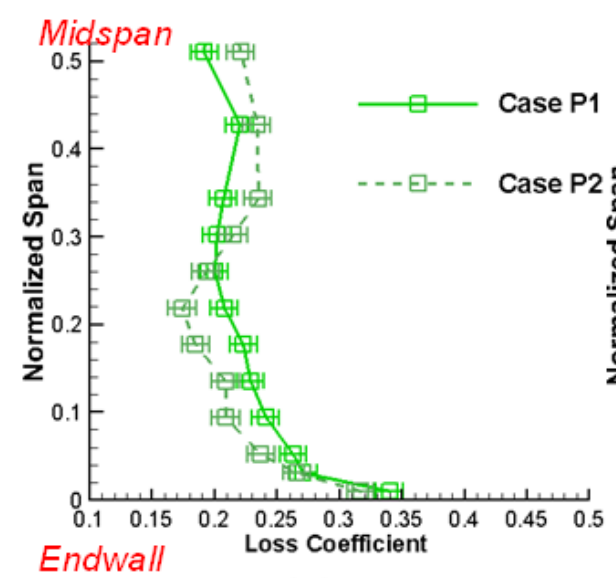

(a)

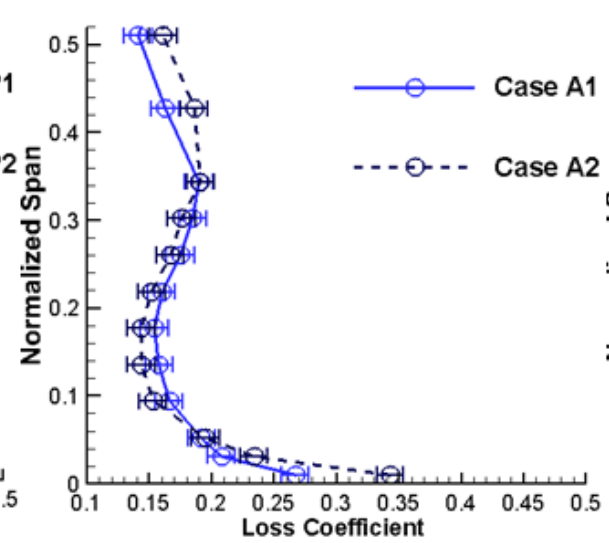

(b)

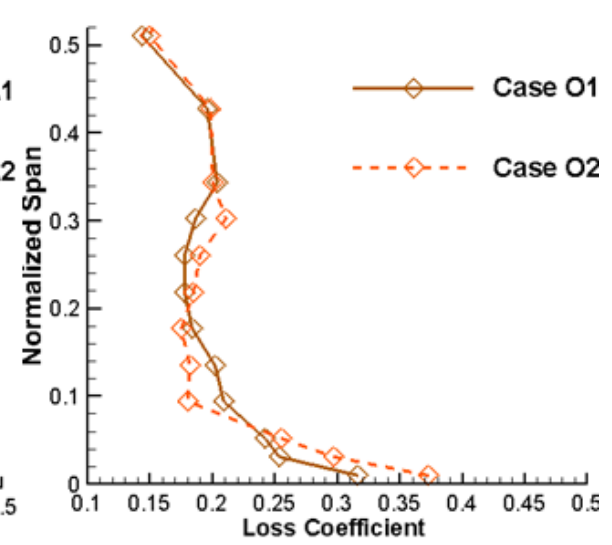

(c)

Figure 24 : Effect of Coolant addition (a) Planar (b) Aero Contour (c) OPT contour - Pitchwise Averaged Loss Coefficients 


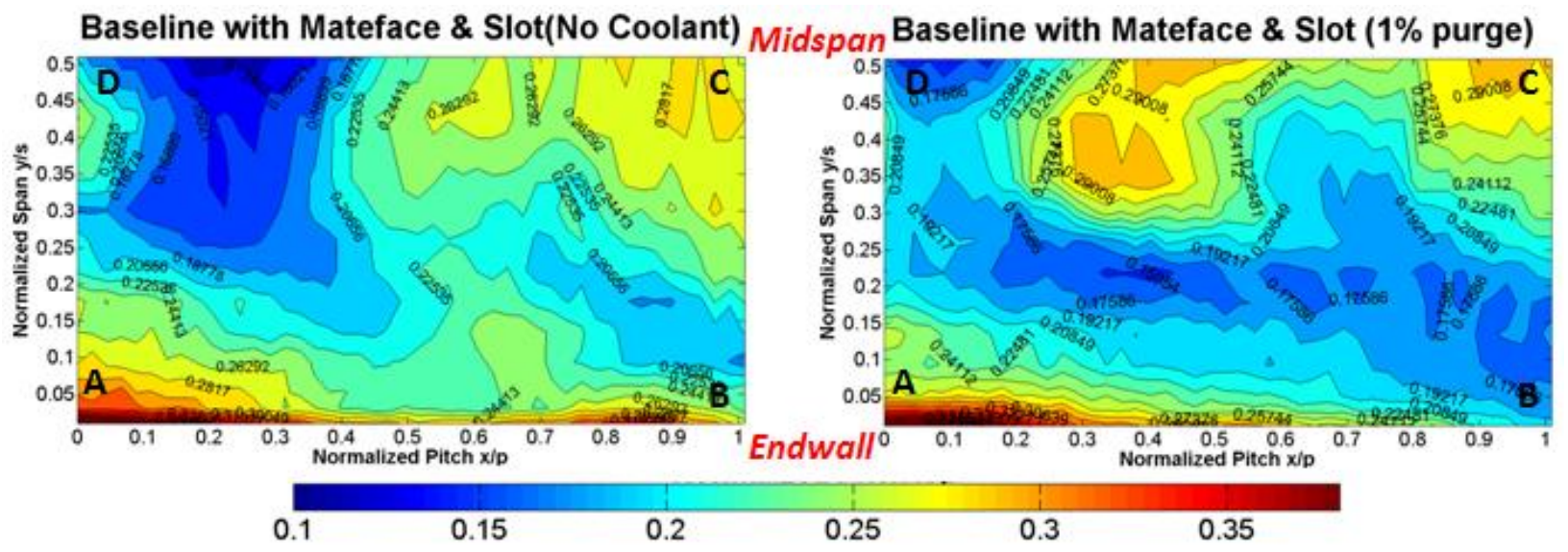

Figure 25 : Effect of Coolant Injection through Upstream Purge Slot in Planar

\section{Endwall Loss Coefficient Contours}

Physically, it seems to suggest that the addition of coolant energizes the boundary layer, which causes the pressure side leg of the horseshoe vortex to travel faster and migrate to the suction side and lift off sooner than what was seen in the without coolant case. This causes higher losses in the region around $35-50 \%$ of the span, contributed by a stronger passage vortex. However reduction in losses from $5-25 \%$ of the span may stem from the fact that the suction side horseshoe vortex continuing down the airfoil is weakened, which is seen in reduced losses near the trailing edge at location(B). The impact of leakage through mateface gap was not investigated in the present study.

\subsection{Effect of Endwall Contouring}

Non axisymmetric endwall contouring is used in gas turbines to modify secondary flow features. The planar endwall is modified by introducing peaks and valleys to weaken the pressure gradient that exists in a high turning passage between airfoils, which can delay the progress of the passage vortex and weaken its intensity, thereby leading to lower losses. The Aero contoured endwall being studied here has a peak region starting near the leading 
edge pressure side that extends towards mid passage and a trough region along the suction side of the airfoil from about 0.2 normalized Cax up to near the trailing edge of the airfoil. The OPT Contour is much flatter in comparison, and discontinuous with gradual troughs and peaks all along.

From the pitchwise averaged loss coefficient plots in Figure 26, it can be seen that the contoured endwalls exhibit lower losses than the planar endwall, both with and without the addition of coolant through the purge slot.

CC

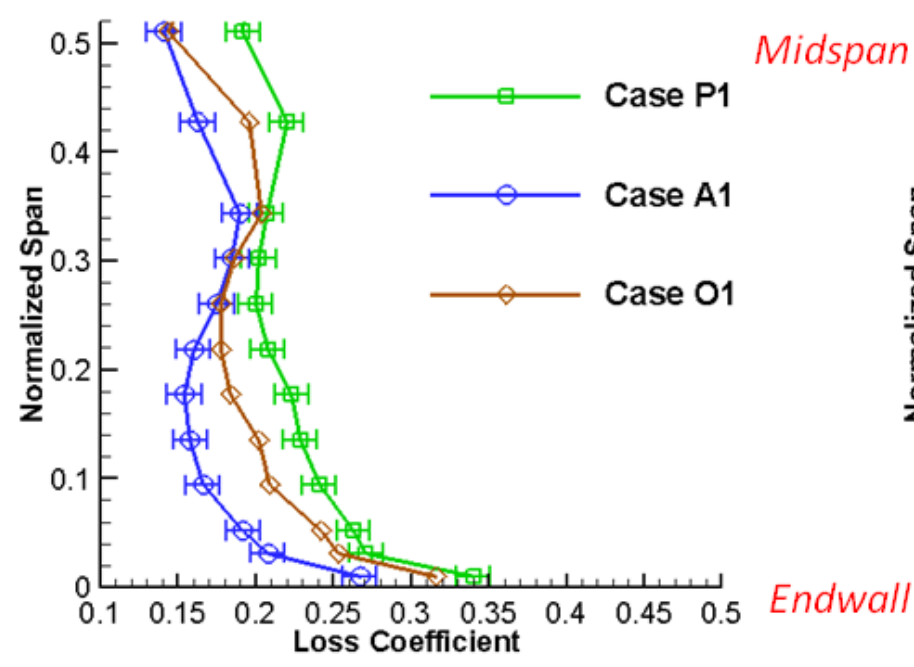

(a)

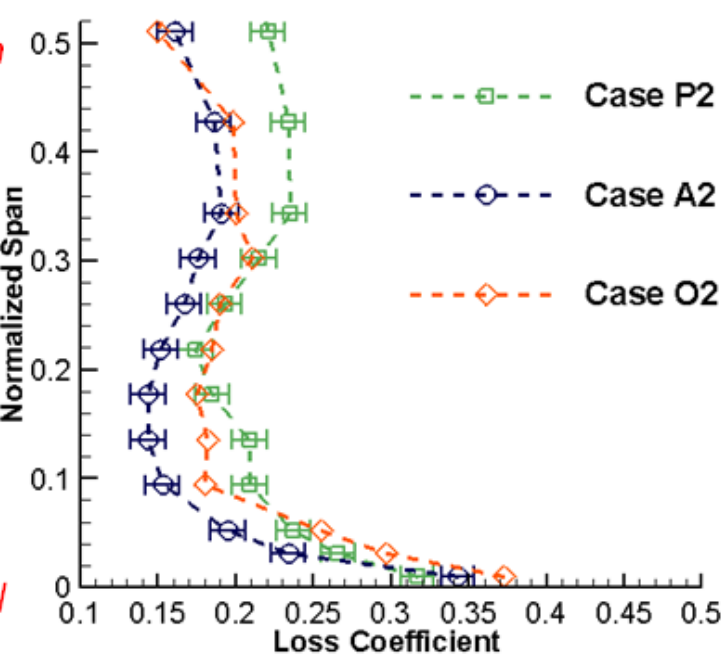

(b)

\section{Figure 26 : Effect of Endwall Contouring - Pitchwise Averaged Loss Coefficients (a) Without Coolant (b) With 1\% MFR through upstream slot}

The Aero contour endwalls seem to be performing the best, and the OPT contour endwall also does better than planar Baseline endwall, both with and without cooling. There is a large reduction in pitchwise averaged losses from about $5-20 \%$ of the span region. For the case with coolant injection $(\mathrm{P} 2, \mathrm{~A} 2, \mathrm{O} 2)$ the difference between the two contoured geometries as compared to the baseline endwall is very less, and within the limit of uncertainty. Some differences exist in the without coolant case(A1 vs O1) from $30-40 \%$ of 
the span. All of these indicate a change in the endwall secondary flow features. It is expected that the contouring changes the distribution of static pressures on the endwall, which subsequently impacts the progression of the passage vortex and the associated vortices induced by it. But these are merely indicative of cases of interest, since the value at each span location has obtained by averaging loss values at all data points across the pitch on that span location. There will be significant pitchwise variation too, owing to the change in secondary flow and these can be better visualized by looking at the whole passage.

The loss contours for the Loss Coefficients for all cases have been plotted in Figure 27. The location of the loss cores near midspan appears to be very similar for both planar and contoured endwall cases. However the magnitude of losses is much higher in the planar cases. The planar endwall shows a region of high concentration of losses near the endwall, particularly near pressure side(A). Loss cores are also seen near midspan which maybe attributed to the mixed out losses from the passage vortex. When compared to the loss coefficient plots for the Aero Contour cases(A1 \& A2), it is seen that the losses overall are much lower, except for a small region near the suction side of the trailing edge(B). The contour is expected to delay the movement of the passage vortex, thereby giving it less time to develop and the passage vortex does not develop in intensity. It was also presented by Panchal [25] using CFD that the Aero contour guides the passage vortex such that it mostly stays closer to the midspan(refer Figure 28), and is much lower in intensity. He also predicted a region of high loss concentration near (B). It is expected that with the addition of mateface, the mainstream flow is ingested in the mateface gap, which is ejected only in the later part. The contour directs this ejected flow towards the suction side trailing edge where it further interacts with the corner vortex. Hence, the flow does not fully diffuse into the mainstream flow, thereby giving higher losses in that location. 
0.1

0.15

0.2

0.25

0.3

0.35

Baseline with Mateface \& Slot(No Coolant) Baseline with Mateface \& Slot (1\% purge)

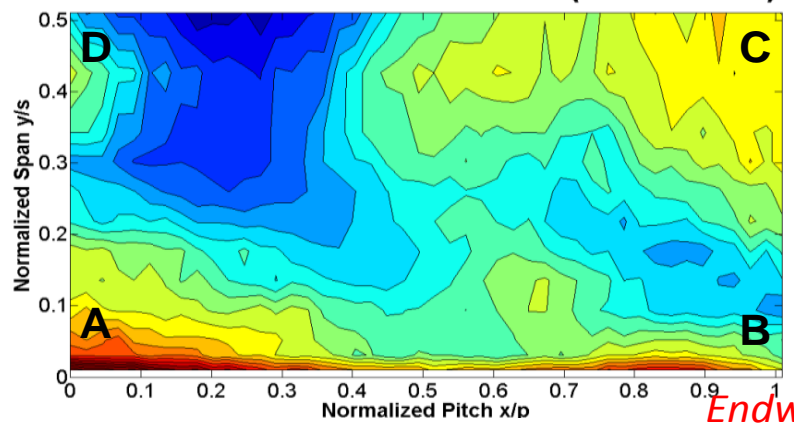

(a) P1

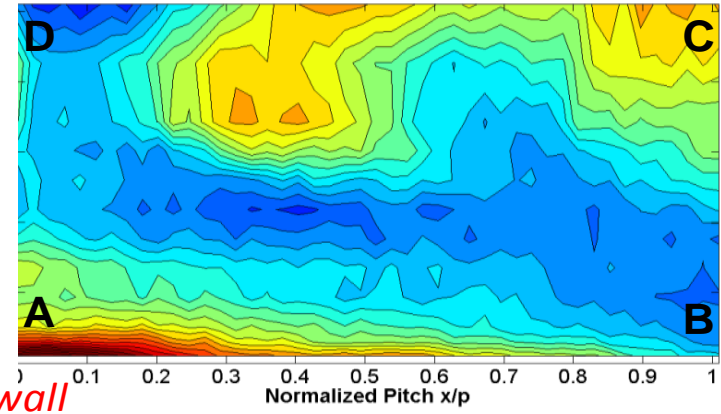

(b) P2

Aero with Mateface \& Slot (No Coolant)

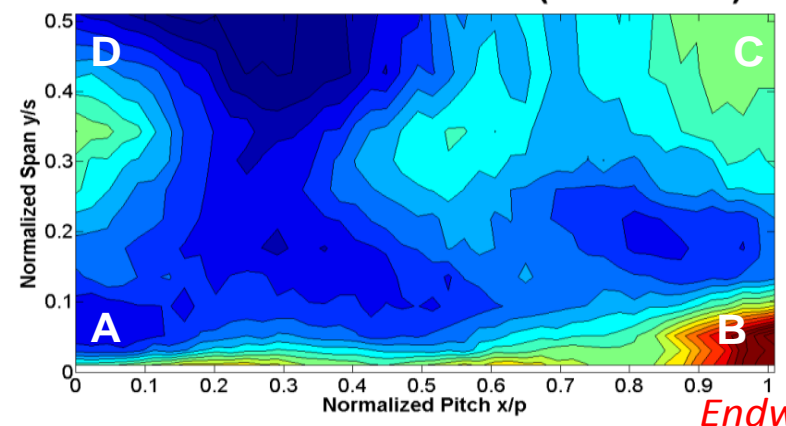

Aero with Mateface \& Slot (1\% purge)

(c) A1

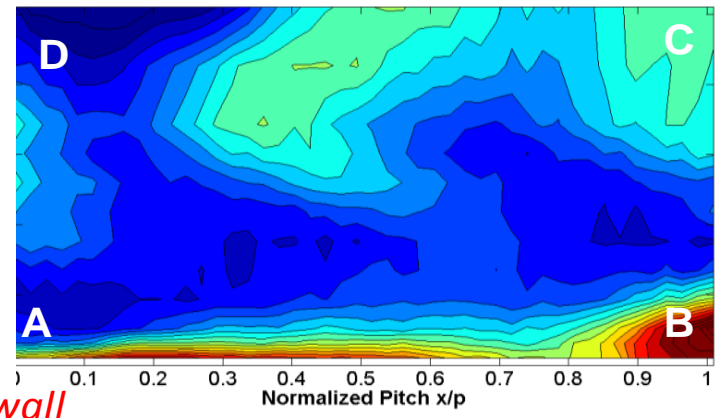

(d) A2

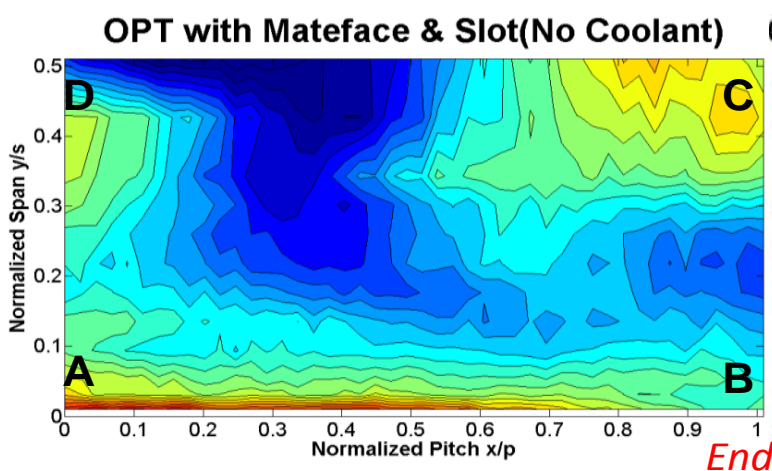

(e) 01

Without Coolant Injection

OPT contour with Mateface \& Slot ( $1 \%$ purge)

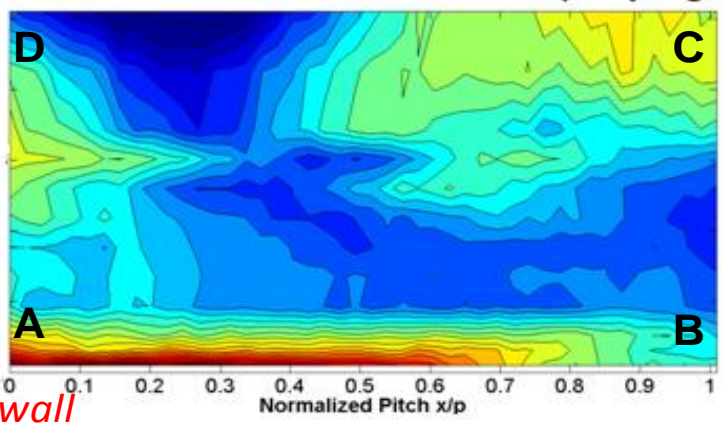

(f) $\mathrm{O} 2$

With 1\% MFR through

Upstream Purge Slot

Figure 27 : Effect of Endwall Contouring with and without Coolant Injection 

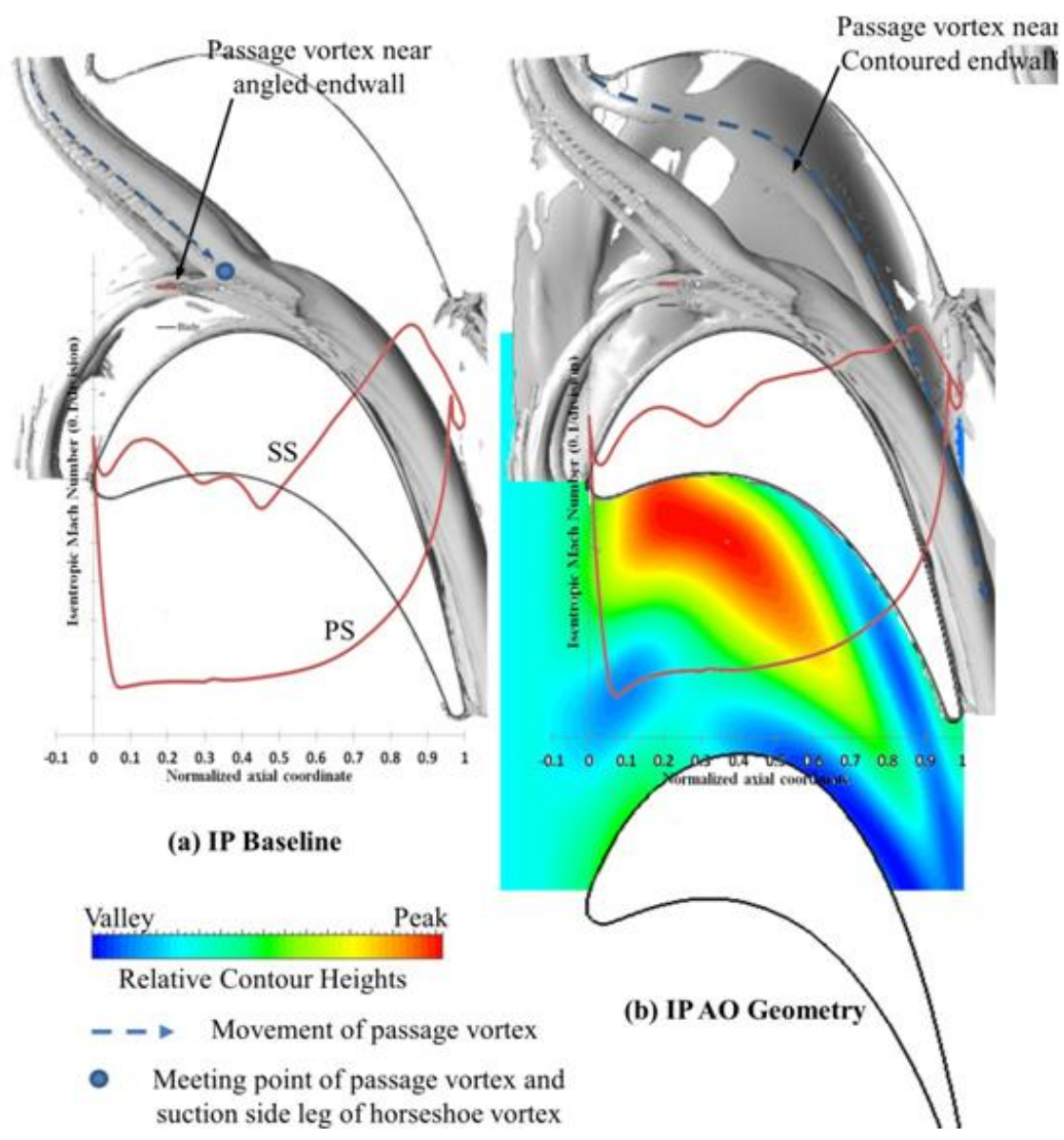

Figure 28 : Comparison of Secondary Flow between Planar endwall and Aero contour [25] (Figure 23 Panchal, K., 2011, "Development of a Robust Numerical Optimization Methodology for Turbine Endwalls and Effect of Endwall Contouring on Turbine Passage Performance,"Doctor of Philosophy, Virginia Polytechnic Institute and State University, Blacksburg, Virginia. Used under fair use, 2013)

As reported in previous studies [21] contoured endwall tries to guide the passage vortex along the pressure side along the airfoil, and delays its progression across the passage. The lift off occurs later, and is lower as compared to the planar endwall. In this loss contour it maybe conjectured that the passage vortex is stronger in case of the planar endwall. Also, since the suction side horseshoe vortex is allowed to continue farther along the airfoil, it develops in strength and maybe responsible for higher losses along the suction side of the trailing edge, depicted in contour plots in corner B. 
The OPT contour(Figure $01 \&$ O2) also shows reduced losses as compared to the planar endwall though the improvement in performance is not as pronounced as the Aero contour. Error! Reference source not found.. The endwall losses are much more spread out, and it is indicative of a higher amount of endwall mixing. This maybe supported by the pitchwise distribution of Exit Mach numbers measured at 1.0 Cax near endwall presented at Figure. It is seen that the Exit Mach numbers for the OPT contour are overall lower throughout the pitch. Even for the baseline and aero cases ( $P 1 \& A 1$ respectively), the higher loss locations on the contour correspond to lower mach numbers, since these locations exhibit higher static pressure due to deceleration of the flow. The higher static pressure generated also supports mateface ingestion. The loss cores near midspan are stronger than the Aero contour case, but seem to be diminished as compared to the planar case. Unlike Aero contour and Baseline, CFD was not available for this contour. Oil flow visualization was performed for the 01 case which confirms that the passage vortex is somewhat delayed. The results for the oil flow will be discussed separately in detail.

\section{Exit Mach\# Near Endwall}

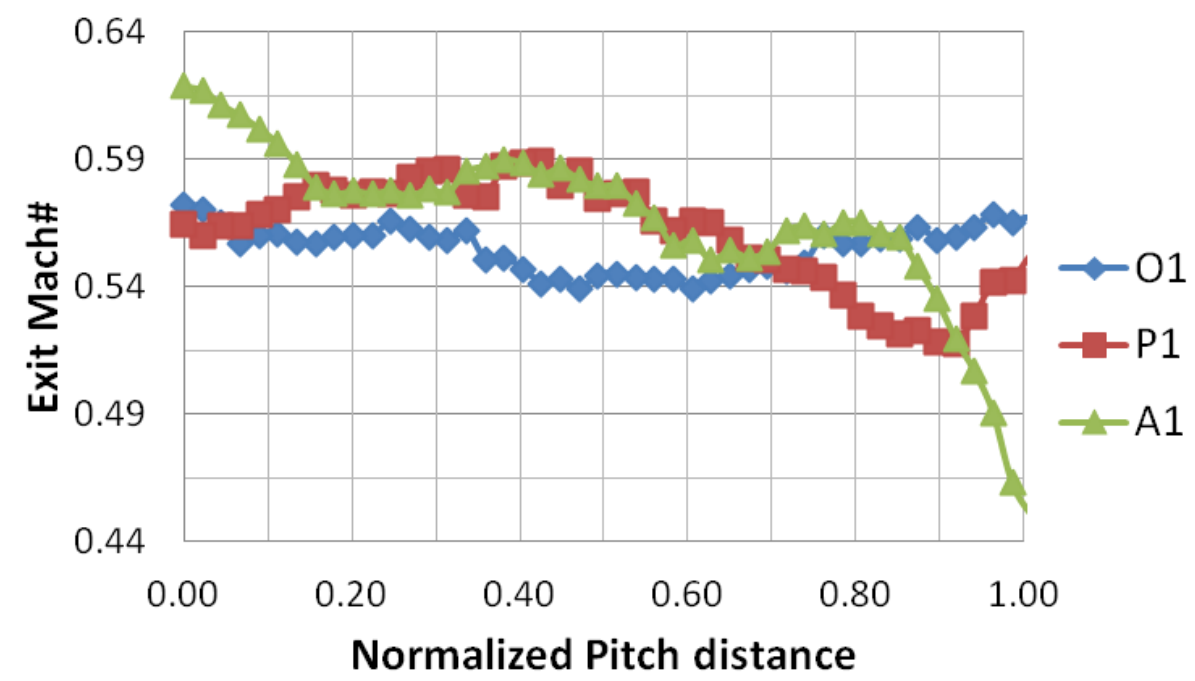

Figure 29 : Near Endwall Exit Mach\# pitchwise distribution for P1,A1 \& 01 42 
Another important aspect of the contouring is that it changes the static pressure profile on the endwall and this has the potential to impact the interaction of coolant with the surface. With the contoured endwall, the pressure gradient on the endwall is much more gradual, as the secondary flow features are guided along the contour, and lesser air is ingested in the mateface. In real time engine operation, this situation does not exist as leakage flow is maintained through the mateface. With a lesser tendency for ingestion in contoured endwall, it may require lower mass flow rates to be injected through the gaps. This could constitute a reduction in parasitic bleed air from the compressor, and may give overall higher efficiency.

Interestingly, the losses for all geometries, i.e. contoured(Aero \& OPT) and planar (Baseline) are relatively similar near endwall in the pitchwise averaged plots; the contoured endwall has higher losses near right trailing edge (B) while the planar endwall has higher loss concentration in left corner(A), thereby pitchwise averages do not differ much. The relative loss distribution in other regions appears to be similar in both endwalls and seem to vary mostly in relative magnitude. The contoured endwall is overall superior to the planar endwall, and this corroborates with previous results by Abraham et al [21].

\subsection{Overall Comparison}

The spanwise plots and contour plots provide a good picture into the overall distribution of losses. However, they do not give a definitive basis for comparison. For this purpose, the overall loss coefficient, as described in equation [2] is used as the metric for comparing overall aerodynamic endwall performance. The planar endwall without coolant case is fixed as the reference case, and the loss coefficient for all other configurations were 
compared to it. All configurations showed reduced losses as compared to the reference case, and the reduction in percentage is tabulated below :

\begin{tabular}{|c|c|c|c|c|}
\hline Endwall Geometry & Coolant & Case & $\begin{array}{l}\text { Loss } \\
\text { Coefficient }\end{array}$ & $\begin{array}{c}\text { Reduction } \\
(\%)\end{array}$ \\
\hline $\begin{array}{l}\text { Baseline Planar Purge } \\
\text { Slot only }\end{array}$ & w/o cooling & P0 & 0.1997 & 10 \\
\hline \multirow{2}{*}{$\begin{array}{c}\text { Baseline Planar } \\
\text { Mateface \& Purge Slot }\end{array}$} & w/o cooling & P1 & 0.2214 & (reference) \\
\hline & $1 \%$ Purge & P2 & 0.2115 & 4 \\
\hline \multirow{2}{*}{$\begin{array}{l}\text { Aero Contour Mateface } \\
\text { \& Purge Slot }\end{array}$} & w/o cooling & A1 & 0.1687 & 24 \\
\hline & $1 \%$ Purge & A2 & 0.1698 & 23 \\
\hline \multirow{2}{*}{$\begin{array}{l}\text { OPT11 Contour } \\
\text { Mateface \& Purge Slot }\end{array}$} & w/o cooling & 01 & 0.1954 & 12 \\
\hline & $1 \%$ Purge & $\mathrm{O} 2$ & 0.1913 & 14 \\
\hline
\end{tabular}

\section{Table 2 : Loss Coefficient Comparison}

For the configuration with planar endwall but no mateface gap, the overall loss coefficient is $10 \%$ lower than the reference case. It can be seen that the contoured endwall shows net reduction in losses as compared to planar, and the addition of coolant causes a slight increase in losses but is still favorably posed. The addition of coolant in planar case shows a slight decrease, however this is inconclusive as this slight reduction falls within limits of experimental uncertainty. The endwall contouring has the maximum impact on reduction of losses as is apparent from the comparison.

The expected shape and location of the passage vortex from the loss contour trends has also been presented in the Figure 30(c). The mateface weakens the passage vortex by breaking the boundary layer, and the effect of contouring as seen for case A1 shall subsequently play an impact on the mixed out losses too. 


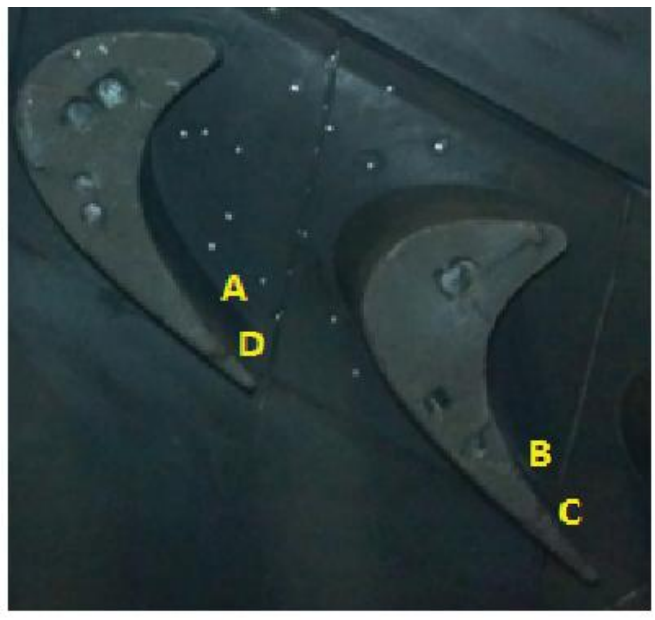

(a)

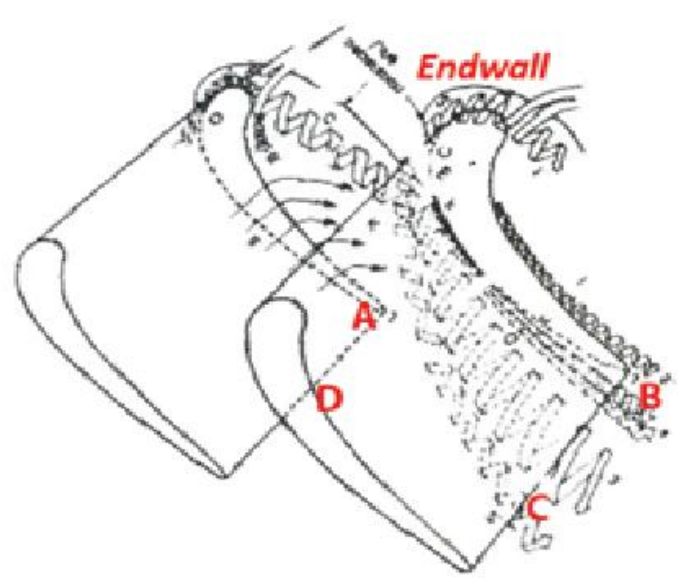

(b)

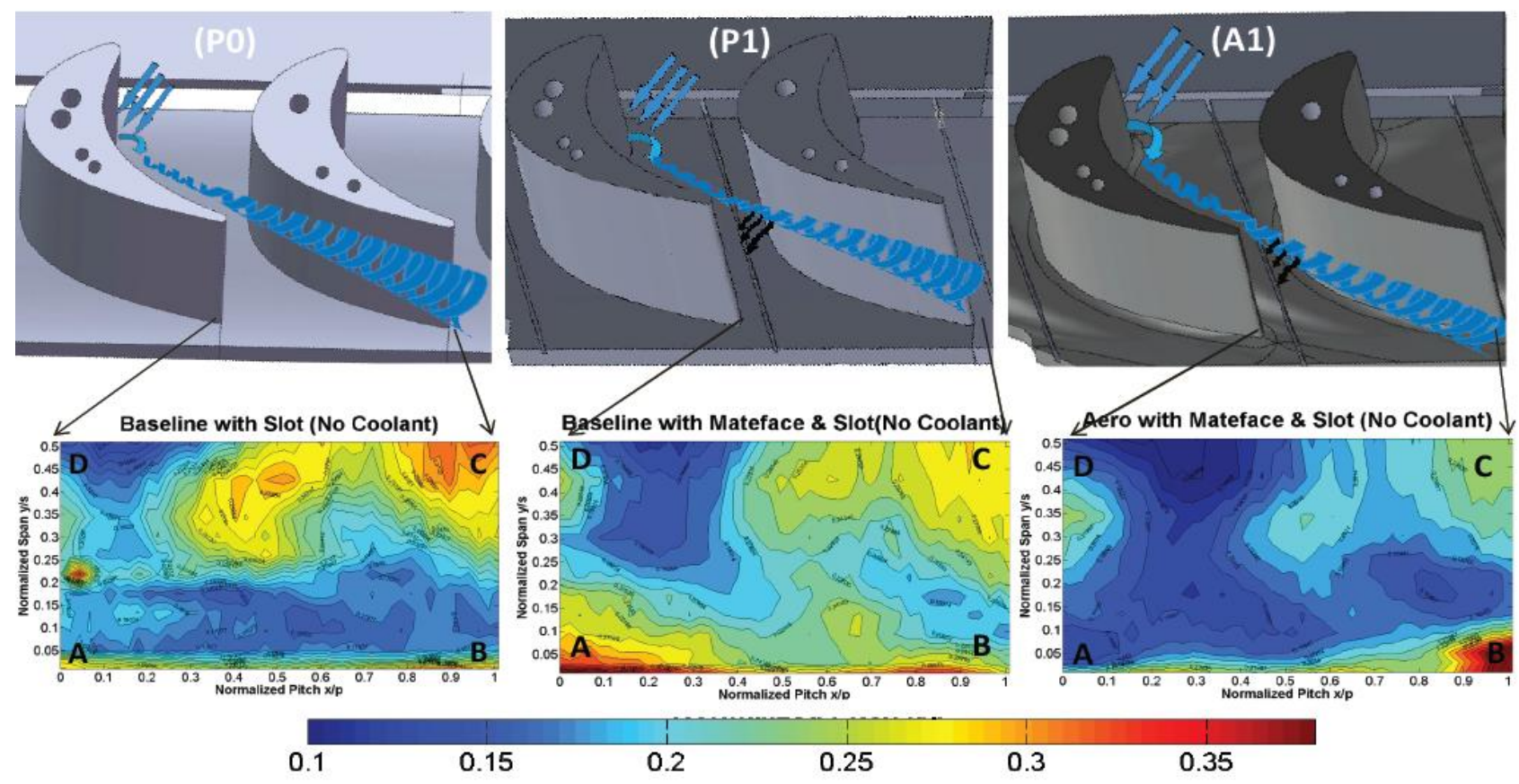

(c)

Figure 30 : (a) Experimental Layout (b) Secondary Flow Structures [11] (c)

Comparison between Loss contours and Passage vortex growth for Planar No Mateface (P0), Planar with Mateface (P1), Contoured with Mateface (C1) in absence of purge cooling Loss Coefficient Contour Plots 


\section{Chapter 5 : Conclusion}

The pressure loss measurements were carried out at 1.0 Cax for a highly loaded, high speed turbine blade at design incidence and design exit Mach number. The effect of endwall contouring, with and without blowing, \& effect of mateface gap have been reported. The lack of data for a no slot geometry with the same experimental conditions does not allow the quantification of the exact amount of change in losses caused by the addition of the upstream slot. This study does give some insight into the incremental loss caused by addition of mateface gap maybe due to ingestion of mainstream flow. However coolant addition has relatively low impact on increment in losses. The major impact on losses in this study has emerged due to endwall contouring. Considering the most realistic case with coolant blowing, at realistic Mach number and turbulence levels, the aerodynamic losses in the form of the overall loss coefficient show a net reduction of $23 \%$ as compared to a reference case of planar endwall with no cooling applied. Contouring changes the overall static pressure profile on the endwall, and makes the gradient less drastic which manifests itself in the diminished intensity of the passage vortex. Additionally, a favorable distribution may also be beneficial to reduce ingestion in mateface gap. This has the potential to impact overall performance since lesser bleed air would be required to avoid mateface ingestion. 


\section{References}

[1] International Energy Outlook 2011 Report Number: DOE/EIA-0484

[2] Soares, C., 2006, "Gas Turbines In Simple Cycle \& Combined Cycle Applications". The Gas Turbine Handbook (National Energy Technology)

[3] Denton, J. D, 1993, "Loss Mechanisms in Turbomachines". J. Turbomach.. $1993 ; 115(4): 621-656$

[4] Hawthorne, W. R. "Rotational Flow Through Cascades Part I. The Components Of Vorticity." The Quarterly Journal of Mechanics and Applied Mathematics 8.3 (1955): 266-279.

[5] Klein, A.,1966, "Investigation of the entry boundary layer on the secondary flows in the blading of axial turbines." BHRA T1004

[6] Sjolander, Andersen, S., 1975, "The endwall boundary layer in an annular cascade of turbine nozzle guide vanes." Carleton University, Department of Mechanical and Aeronautical Engineering,

[7] Langston, L. S.,1980, "Crossflows in a turbine cascade passage." American Society of Mechanical Engineers, Gas Turbine Conference and Products Show, New Orleans, La.

[8] Sieverding, C. H.,1985, "Recent progress in the understanding of basic aspects of secondary flows in turbine blade passages." Journal of Engineering for Gas Turbines and Power $107.2: 248-257$.

[9] Sharma, O. P., Butler T. L., 1987, "Predictions of endwall losses and secondary flows in axial flow turbine cascades." Journal of turbomachinery 109.2: 229-236. 
[10] Goldstein, R. J., Spores, R. A., 1988, "Turbulent transport on the endwall in the region between adjacent turbine blades." Journal of Heat Transfer (Transcations of the ASME, Series C);(United States) 110.4A

[11] Takeishi, K., 1989, "An experimental study of heat transfer and film cooling on low aspect ratio turbine nozzles." American Society of Mechanical Engineers

[12] S. Acharya,2000, "Endwall Cooling With Endwall Contouring and Leading Edge Fillet," Semi-annual Report Submiited to UTSR, South Carolina, Project No. 02-01SR098, June 2003-December 2003; D.E. Bohn, K. Kusterer, N. Sürken, and F.Kreitmeler, "Influence of Endwall Contouring in Axial Gaps on the Flow Field in a Four-Stage Turbine," ASME Proc.Turbo Expo, 2000-GT-472, 2000; L.P. Timko, "Energy Efficient Engine High Pressure Turbine Component Test Performance Report," Contract Report for NASA, Report No. NASA CR-168289

[13] Blair, M.F., 1974, "An Experimental Study of Heat Transfer and Film Cooling on Large-Scale Turbine Endwalls", ASME J. Heat Transfer, 96, pp. 524-529.

[14] de la Rosa Blanco, E., Hodson, H.P., and Vazquez, R., 2006, "Effect of the leakage flows and the upstream platform geometry on the endwall flows of a turbine cascade", ASME Paper No. GT2006-90767.

[15] Hada, S., and Thole, K. A., 2006, "Computational Study of a Midpassage Gap and Upstream Slot on Vane Endwall Film-Cooling," ASME Paper No. GT2006-91067.

[16] Piggush, J. D., and Simon, T. W., 2005, "Flow Measurements in a First-Stage Nozzle Cascade Having Endwall Contouring, Leakage and Assembly Features," ASME Paper No. GT2005-68340.

[17] Piggush, J. D., and Simon, T. W, 2005, "Flow Measurements in a First StageNozzle Cascade Having Leakage and Assembly Features: Effects of 
EndwallSteps and Leakage on Aerodynamic Losses" ASME Paper No. IMECE200583032.

[18] Yamao, H., Aoki, K., Takeishi, K., and Takeda, K., 1987, “An Experimental Study for Endwall Cooling Design of Turbine Vanes," IGTC-1987, Tokyo, Japan.

[19] Reid, K., Denton, J., Pullan, G., Curtis, E., and Longley, J., 2007, "The Interaction of Turbine Inter-Platform Leakage Flow With the Mainstream Flow," ASME J. Turbomach., 129, pp. 303-310.

[20] Kost, F., \& Nicklas, M.,2001, "Film-cooled turbine endwall in a transonic flow field: Part I-Aerodynamic measurements". Journal of turbomachinery, 123(4), 709-729.

[21] Abraham, S., Panchal, K., Ekkad, S. V., Ng, W. F., Lohaus, A. S., and Malandra, A., 2012, "Effect of Endwall Contouring on a Transonic Turbine Blade Passage: Part 1 - Aerodynamic Performance", ASME Paper No. GT2012-68425.

[22] Blot, D., Roy, A., Ekkad, S. V., Ng, W., Lohaus, A. S., and Crawford, M. E., 2013, "Effect of upstream purge slot on a transonic turbine blade passage: Part 1 Aerodynamic Performance ", ASME Paper No. GT2013-94591.

[23] Panchal, K.V., Abraham, S., Ekkad, S.V., Ng, W.F., Brown, B., and Malandra, A., 2011, "Investigation of effect of endwall contouring methods on a transonic turbine blade passage", ASME Paper No.GT2011-45192.

[24] Kline, S.J., and McClintock, F.A., 1953, "Describing uncertainties in single sample experiments", Mechanical Engineering, pp. 3-8.

[25] Panchal, K., 2011, "Development of a Robust Numerical Optimization Methodology for Turbine Endwalls and Effect of Endwall Contouring on Turbine Passage Performance," Doctor of Philosophy, Virginia Polytechnic Institute and State University, Blacksburg, Virginia. 
[26] Acharya, S, Mahmoood., G, 2006, "Turbine Blade Aerodynamics". The Gas Turbine Handbook Chapter 4.3 (National Energy Technology).

[27] Han, J. C., Datta, S., \& Ekkad, S. (2013). Gas turbine heat transfer and cooling technology. CRC Press. 